\title{
Measurable Multiattribute Value Functions for Portfolio Decision Analysis
}

\author{
Juuso Liesiö \\ Department of Mathematics and Systems Analysis, Aalto University, P.O. Box 11100, 00076 Aalto, Finland \\ juuso.liesio@aalto.fi
}

Portfolio decision analysis models support selecting a portfolio of projects in view of multiple objectives and limited resources. These models often rely on the additive-linear portfolio value function, although empirical evidence suggests that the underlying preference assumptions do not always hold. In this paper we relax these assumptions and derive a more general class of portfolio value functions which deploy symmetric multilinear functions to capture non-linearities in the criterion specific portfolio values. These criterion specific portfolio values can be aggregated with an additive, a multiplicative or a multilinear function allowing a rich representation of preferences among the multiple objectives. We develop novel techniques for eliciting these value functions and also discuss the use of existing techniques that are often applied in practice. Furthermore, we demonstrate that the value functions can be maximized for problem sizes of practical relevance using an implicit enumeration algorithm or an approximate mixed integer linear programming model. Application of the results is illustrated with an example in ecological conservation site selection.

Key words: multiattribute value theory, project portfolio selection, resource allocation

\section{Introduction}

Selecting which portfolio of project candidates (e.g., products, infrastructure investments, research programmes, policy options) to implement with the available resources is an important decision problem both in public administration and private companies (e.g., Golabi et al. 1981, Ewing Jr. et al. 2006, Liesiö et al. 2007, Kleinmuntz 2007, Phillips and Bana e Costa 2007, Grushka-Cockayne et al. 2008). Maximizing the value of a project portfolio is often complicated by multiple value criteria and a large number of alternative portfolios. Plenty of applied and theoretical research has been done to develop analytic support for such decisions, mainly under titles such as portfolio decision analysis, project portfolio selection and resource allocation (for overviews see, e.g., Klein- 
muntz 2007, Salo et al. 2011). Common to most of this work is the use of multiattribute value or utility functions to model preferences over alternative resource allocations or portfolios, and the deployment of mathematical optimization techniques to identify the most preferred portfolio subject to resource and other portfolio constraints.

The additive-linear portfolio value function (Golabi et al. 1981), in which portfolio value is the sum of additive multiattribute project values, has become a standard in multiobjective portfolio decision analysis applications. Often its use is motivated by the simple preference elicitation process and computational tractability: Preferences are captured by criterion specific project value functions and criterion weights, and the optimal portfolio is obtained by solving an integer linear programming (ILP) problem. However, the additive-linear portfolio value function assumes that the value increase resulting from adding a project into the portfolio does not depend on what other projects are included in the portfolio. This assumption does not often hold in practice: For instance, Kleinmuntz (2007) reports experiences in health-care capital budgeting cases, where improvements in the portfolio's aggregate financial return become less important relative to improvements in other attributes, when the aggregate financial return increases. Mild and Salo (2009) note that in infrastructure asset portfolio management, the marginal value of improving assets' quality class may decrease, when the number of assets in the best quality class increases.

The literature offers very few tools to cope with non-linear criterion specific portfolio value. For monetary attributes (e.g., cash-flow), the sum of the projects' performances (e.g., net cash-flow) is often a natural measure of portfolio performance, in which case a non-linear value function over the sum can be used to capture non-constant marginal value (e.g., Argyris et al. 2011) or threshold constraints can be introduced to limit the sum into a range where the marginal value is approximately constant (e.g., Kleinmuntz 2007). However, these approaches are not directly applicable for 'non-additive' attributes or attributes without a natural measurement scale, which both are common in applications of multiobjective portfolio decision analysis (e.g., Golabi et al. 1981, Ewing Jr. et al. 2006, Liesiö et al. 2007, Kleinmuntz 2007, Phillips and Bana e Costa 2007). As an exception, Grushka-Cockayne et al. (2008) use non-additive criterion specific portfolio value functions in the 
context of development project selection, but do not discuss the underlying preference assumptions in detail.

In this paper we develop non-additive portfolio value functions based on the theory of measurable multiattribute value functions by Dyer and Sarin (1979). We show that project-symmetric preferences and weaker preference independence assumptions than those underlying the additivelinear portfolio value imply that the projects' criterion specific values are combined with symmetric multilinear functions. The resulting criterion specific portfolio values are then aggregated with an additive, a multiplicative or a multilinear function. We develop techniques to elicit these value functions, derive several special forms of them and illustrate how they relate to the standard additive-linear portfolio value. Both exact and approximate integer programming models are developed for maximizing these portfolio value functions subject to linear constraints.

The rest of this paper is structured as follows. Section 2 introduces the problem setting with an illustrative example in ecological conservation site selection. Section 3 establishes the formal preference assumptions underlying the additive-linear model. Section 4 relaxes some of these assumptions, derives the corresponding portfolio value functions and develops techniques for eliciting these functions. Section 5 presents optimization models for maximizing the developed portfolio value functions. Section 6 applies these results to the example of Section 2. Section 7 discusses some practical aspects of these models and Section 8 concludes.

\section{Preliminary example}

We begin with an illustrative example to introduce the standard framework for multiobjective portfolio decision analysis by Golabi et al. (1981). The example is based on pilot projects carried out in Finland in which public funds were used to purchase privately owned forest sites for conservation for a fixed period of some 10-20 years. In many of these projects the public officials used an additive multiattribute value function over relevant conservation criteria to form a priority order in which the offered sites were acquired for conservation. Here we present a stylized version of this problem in which the public officials seek to maximize the conservation value of a site portfolio subject to a limited budget. 
Table 1 Criteria and measurement scales

\begin{tabular}{c|ccccc}
\hline$i$ & Criterion name & Measurement unit & $x_{i}^{0}$ & $x_{i}^{*}$ & $X_{i}$ \\
\hline 1 & Area & ha & 0.5 & 5 & {$[0.5,5]$} \\
2 & Old broad-leaved trees & $\mathrm{m}^{3}$ & 0 & 200 & {$[0,200]$} \\
3 & Natural water economy & verbal & none & excellent & $\{$ none, poor, good,excellent $\}$ \\
4 & Endangered species & number & 0 & 100 & $\{0,1, \ldots, 100\}$ \\
5 & Closest nature reserve & $\mathrm{km}$ & 50 & 0 & {$[0,50]$}
\end{tabular}

The sites (cf. projects) are evaluated with regard to $n=5$ criteria indexed $i \in I=\{1, \ldots, n\}$ whose measurement scales $X_{i}$ and the most and the least preferred performance levels $\left(x_{i}^{*}\right.$ and $x_{i}^{0}$, respectively) are presented in Table 1 . The performance of any site is captured by a row-vector in $X_{1} \times \ldots \times X_{n}$ (e.g., $\left(3 \mathrm{ha}, 100 \mathrm{~m}^{3}\right.$, poor $\left.\left., 80,10 \mathrm{~km}\right)\right)$ and $\left(x_{1}^{0}, \ldots, x_{n}^{0}\right)$ is the base-line performance that results if a site is not selected (in general the base-line can be any performance in $X_{1} \times \ldots \times X_{n}$; Golabi et al. 1981, Clemen and Smith 2009). Hence, the performance of a site portfolio is captured by a matrix $x$ with $m$ rows denoted by $x_{j I}=\left(x_{j 1}, \ldots, x_{j n}\right), j \in J=\{1, \ldots, m\}$. Here $m$ does not have to be equal to the number of actual site candidates available, but defines the maximum number of site candidates in the value model (cf. choosing the end points for the measurement scales in multiattribute models; see, e.g., von Winterfeldt and Edwards 1986).

A portfolio value function is a mapping $V: X \rightarrow \mathbb{R}$, where $X=\left\{X_{1} \times \ldots \times X_{n}\right\}^{m}$ is the $m \cdot n$ dimensional space of all possible portfolio performances $x$. The additive-linear portfolio value function is $V(x)=\sum_{i=1}^{n} w_{i}(1) \sum_{j=1}^{m} v_{i}\left(x_{j i}\right)$ (Golabi et al. 1981), where $v_{i}: X_{i} \rightarrow[0,1]$ are the criterion specific site value functions and $w_{i}(1)$ is the weight of criterion $i$; it captures the value increase when the criterion specific performance of a single site is changed from the least preferred level $x_{i}^{0}$ to the most preferred level $x_{i}^{*}$. The criterion specific site value functions used in the example are presented in Figure 1.

To formulate the portfolio optimization problem we introduce binary decision variables $z_{1}, \ldots, z_{m}$, such that $z_{j}=1$ if site $j$ is selected and $z_{j}=0$ if it is not selected. The set of feasible decision variable values is $Z_{F}=\left\{z \in\{0,1\}^{m} \mid A z \leq B\right\}$, where matrix $A \in \mathbb{R}^{q \times m}$ and vector $B \in \mathbb{R}^{q}$ code the $q$ linear feasibility constraints (see, e.g., Liesiö et al. 2007). The limited budget for acquiring sites for conservation is modeled by a single constraint $(q=1)$ with $B$ equal to the total budget and rowvector $A$ containing the cost of each site. With the additive-linear $V$ the most preferred portfolio 

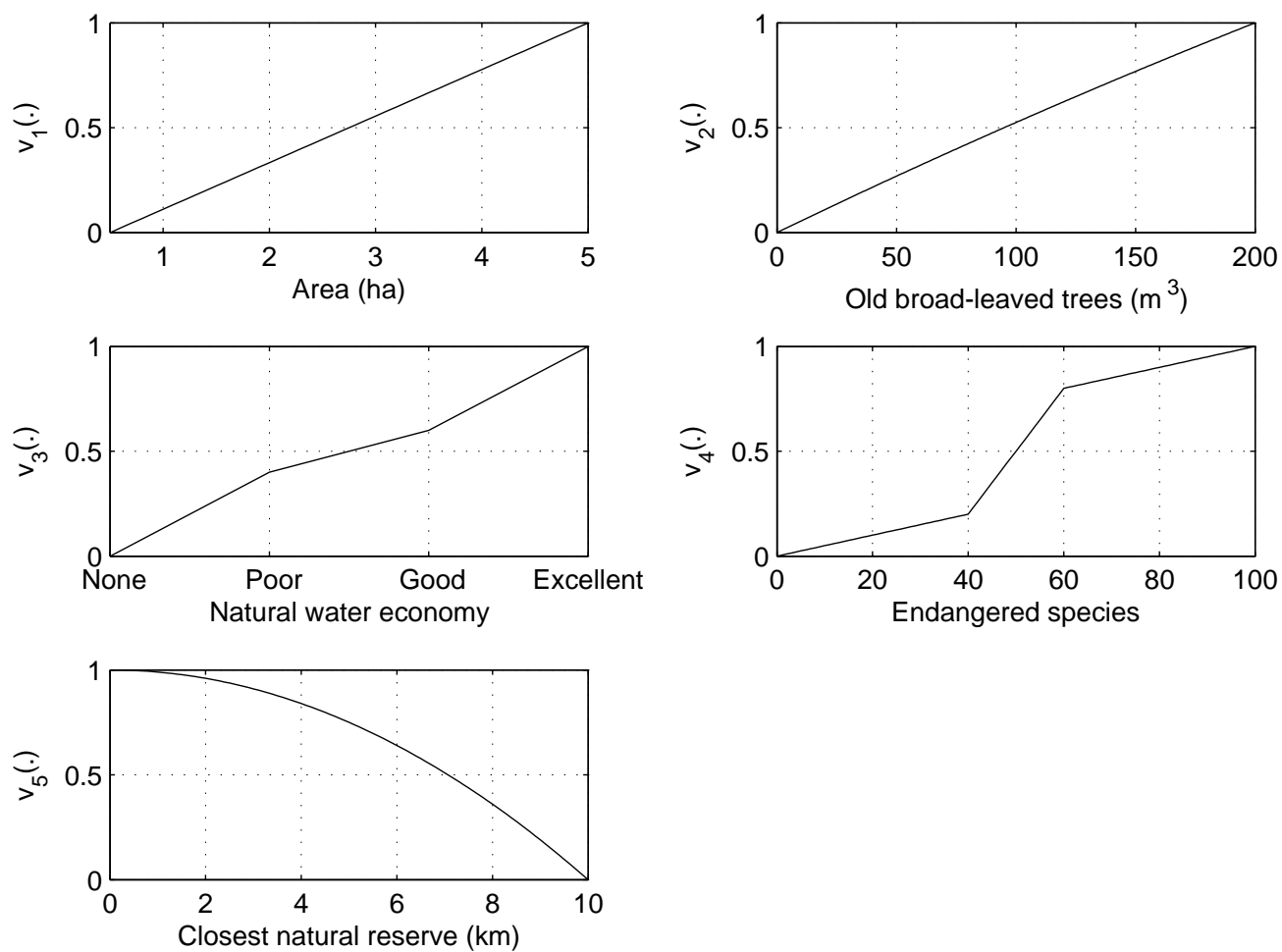

Figure 1 Criterion specific project value functions $v_{1}, \ldots, v_{5}$.

is obtained from the ILP problem $\max _{z \in Z_{F}} \sum_{i=1}^{n} w_{i}(1) \sum_{j=1}^{m}\left[z_{j} v_{i}\left(x_{j i}^{g o}\right)+\left(1-z_{j}\right) v_{i}\left(x_{j i}^{n o}\right)\right]$, where $x_{j i}^{g o}$ and $x_{j i}^{n o}$ denote the performances when the $j$ th site is selected and not selected, respectively (i.e., in this example $x_{j i}^{n o}=x_{i}^{0}$ for all $\left.j \in J, i \in I\right)$.

The additive-linear value function does not allow non-constant marginal value; adding a project into the portfolio results in the same value increase independent of the composition of the portfolio. For instance, when considering a single site the DM may state that criterion 'Natural water economy' $(i=3)$ is more important than 'Endangered species' $(i=4)$ in the sense that $w_{3}(1)>w_{4}(1)$. The additive-linear value function then implies that a site with performances $\left(y_{1} \mathrm{ha}, y_{2} \mathrm{~m}^{3}\right.$, exc., $\left.0, y_{5} \mathrm{~km}\right)$ adds more value than a site with performances ( $y_{1}$ ha, $y_{2} \mathrm{~m}^{3}$, none, $\left.100, y_{5} \mathrm{~km}\right),\left(y_{1}, y_{2}, y_{5}\right) \in X_{1} \times X_{2} \times X_{5}$, even when the portfolio contains a lot of other sites with excellent natural water economy but few endangered species. 


\section{Preference Assumptions Underlying the Additive-Linear Portfolio Value}

In this section we establish the four preference assumptions underlying the additive-linear portfolio value function and in Section 4 relax the last one of these to derive a more general class of portfolio value functions. We use the framework of measurable value functions by Dyer and Sarin (1979), but in Section 7 discuss how corresponding results can be obtained with multiattribute utility functions (Keeney and Raiffa 1976). The use of measurable value functions requires that the DM's preferences are captured by weak orders $\succeq$ and $\succeq_{D}$ on $X$ and $X \times X$, respectively; $x^{a} \succeq x^{b}$ denotes that portfolio $x^{a}$ is (equally) preferred to portfolio $x^{b}$ and $\left(x^{a} \leftarrow x^{b}\right) \succeq_{D}\left(x^{c} \leftarrow x^{d}\right)$ denotes that the change from portfolio $x^{b}$ to portfolio $x^{a}$ is (equally) preferred to the change from portfolio $x^{d}$ to portfolio $x^{c}$. Equal preferences $\left(\sim, \sim_{D}\right)$ and strict preferences $\left(\succ, \succ_{D}\right)$ among portfolios and changes are defined in a standard way. A measurable portfolio value function $V: X \rightarrow \mathbb{R}$ represents these preference orders in the sense that for any portfolios $x^{a}, x^{b}, x^{c}, x^{d} \in X$

$$
\begin{gathered}
V\left(x^{a}\right) \geq V\left(x^{b}\right) \Leftrightarrow x^{a} \succeq x^{b}, \\
V\left(x^{a}\right)-V\left(x^{b}\right) \geq V\left(x^{c}\right)-V\left(x^{d}\right) \Leftrightarrow\left(x^{a} \leftarrow x^{b}\right) \succeq_{D}\left(x^{c} \leftarrow x^{d}\right) .
\end{gathered}
$$

$V$ is unique up to positive affine transformations: if $V^{\prime}=\alpha V+\beta$ for some $\alpha>0$, and $\beta \in \mathbb{R}$ then both $V$ and $V^{\prime}$ imply the same preferences $\succeq$ and $\succeq_{D}$. Hence, for the rest of the paper we scale the portfolio value such that $V\left(x^{0}\right)=0$ and $V\left(x^{*}\right)=1$, where $x^{0}$ and $x^{*}$ denote the least and most preferred portfolios, respectively. Since $V: X \rightarrow[0,1]$ can be interpreted as an $m \cdot n$-attribute value function we refer to the terms $X_{j i}$ of the cartesian product $X=\times_{j \in J, i \in I} X_{j i}$ as attributes. However, the same set of $n$ criteria is used to evaluate all projects and hence the measurement scale for each attribute $X_{j i}$ is $X_{i}$. Figure 2 illustrates the attribute structure as a value tree.

The first preference assumption is that two portfolio performances $x^{a}$ and $x^{b}$ that contain exactly the same project performances are equally preferred even if these performances are not produced by the same projects. More specifically, if by changing the order of rows of $x^{a}$ it is possible to obtain matrix that is equal to $x^{b}$, then $x^{a} \sim x^{b}$. In the site selection example this assumption would imply, for instance, that 


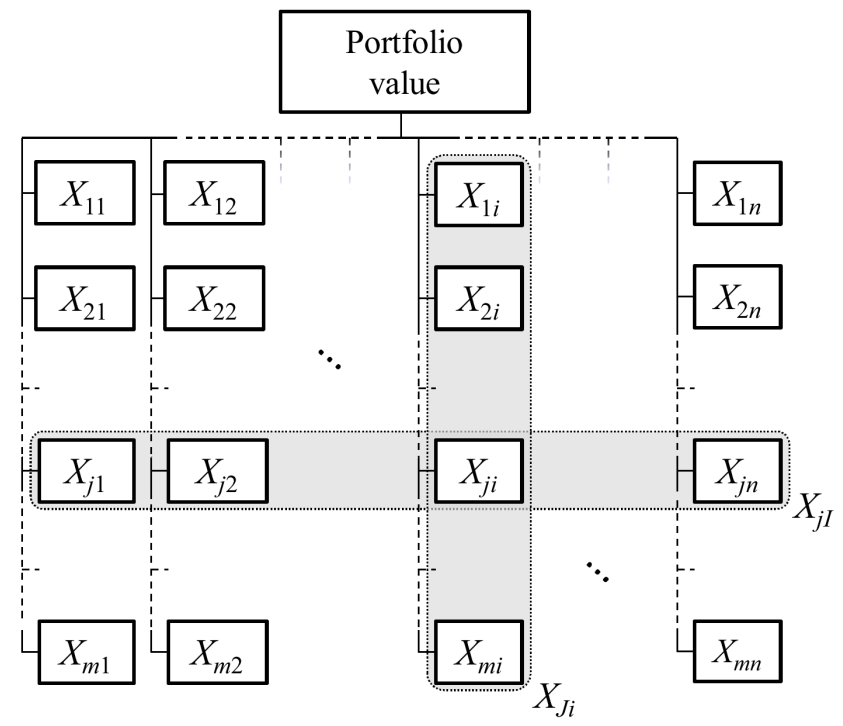

Figure 2 The value tree consisting of $m \cdot n$ (twig-level) attributes $X_{j i}$. The measurement scales are identical in each column, i.e., $X_{j i}=X_{i}$ for all $j \in J, i \in I$.

$$
x^{a}=\left(\begin{array}{ccccc}
3 \mathrm{ha} & 100 \mathrm{~m}^{3} & \text { none } & 80 & 10 \mathrm{~km} \\
0.5 \mathrm{ha} & 0 \mathrm{~m}^{3} & \text { none } & 0 & 50 \mathrm{~km} \\
0.5 \mathrm{ha} & 0 \mathrm{~m}^{3} & \text { none } & 0 & 50 \mathrm{~km} \\
\vdots & \vdots & \vdots & \vdots & \vdots
\end{array}\right) \sim\left(\begin{array}{ccccc}
0.5 \mathrm{ha} & 0 \mathrm{~m}^{3} & \text { none } & 0 & 50 \mathrm{~km} \\
3 \mathrm{ha} & 100 \mathrm{~m}^{3} & \text { none } & 80 & 10 \mathrm{~km} \\
0.5 \mathrm{ha} & 0 \mathrm{~m}^{3} & \text { none } & 0 & 50 \mathrm{~km} \\
\vdots & \vdots & \vdots & \vdots & \vdots
\end{array}\right)=x^{b} .
$$

Assumption 1. The preferences $\succeq$ are project-symmetric, i.e., any portfolio performances $x^{a}, x^{b} \in$ $X$ that are equal up to permutation of rows are equally preferred.

The rest of the preference assumptions make use of the concept of weak difference independence of attribute sets (Dyer and Sarin 1979). A set of attributes is said to be weak difference independent (WDI) if the preferences among changes $\left(\succeq_{D}\right)$ in the performance levels of these attributes are the same for any performance levels of the other attributes (see Appendix A for mathematical details). If a set of attributes is WDI, then it is also preference independent (PI), i.e., preferences among the performance levels $(\succeq)$ of these attributes do not depend on the performance levels of the other attributes (Dyer and Sarin 1979).

Preferences $\succeq_{D}$ among changes in the criterion specific performance level of a single project are assumed independent of (i) the performance of the project w.r.t. other criteria and (ii) the performances of the other projects. This assumption is a pre-requisite for eliciting criterion specific 
project value functions (project scoring); for instance, making the statement $40 \leftarrow 0 \sim_{D} 100 \leftarrow 60$ regarding the value of endangered species on a site (see $v_{4}$ in Figure 1 ) requires that the level of the other attributes does not matter.

Assumption 2. Each attribute $X_{j i}, j \in J, i \in I$, is WDI.

Assumptions 1 and 2 ensure that there exist most $x_{i}^{*}$ and least $x_{i}^{0}$ preferred levels on each measurement scale $X_{i}$ such that the most and least preferred portfolios $x^{*}$ and $x^{0}$ satisfy $x_{j i}^{*}=x_{i}^{*}$ and $x_{j i}^{0}=x_{i}^{0}$ for all $i \in I, j \in J$. In the site selection example the most and least preferred portfolios consist of $m$ sites with performances $\left(5 \mathrm{ha}, 200 \mathrm{~m}^{3}\right.$, exc., $\left.100,0 \mathrm{~km}\right)$ and $\left(0.5 \mathrm{ha}, 0 \mathrm{~m}^{3}\right.$, none, $\left.0,10 \mathrm{~km}\right)$, respectively.

The third assumption is that preferences $\succeq_{D}$ over attributes that measure the criterion specific portfolio performance do not depend on the portfolio's performance w.r.t. all other attributes. This means that each criterion can be used as a meaningful measure of portfolio performance by examining how projects in the portfolio perform w.r.t. the criterion in question. In the site selection example this implies, for instance, that the DM can state if the change $(\text { exc., none }, \ldots, \text { none })^{T} \leftarrow(\text { none }, \ldots, \text { none })^{T}$ in the water economy criterion $(i=3)$ is preferred to the change (good, good, none, $\ldots$, none $)^{T} \leftarrow(\text { none }, \ldots, \text { none })^{T}$ without knowing the fixed levels of the other attributes $X_{j i}, j \in J, i \neq 3$.

AsSUMPTION 3. Each set of attributes measuring the criterion specific portfolio performance $X_{J i}=$ $X_{1 i} \times \cdots \times X_{m i}, i \in I$, is WDI.

Assumptions 1-3 hold throughout the paper, but the one additional assumption necessary for the additive-linear value function will be relaxed later. This is that preferences $\succeq_{D}$ over the performance levels of a single project are independent of the performances of other projects in the portfolio. In our example this assumption implies, for instance, that the preferences between of site performances $\left(0.5 \mathrm{ha}, 0 \mathrm{~m}^{3}\right.$, exc., $\left.0,10 \mathrm{~km}\right)$ and $\left(0.5 \mathrm{ha}, 0 \mathrm{~m}^{3}\right.$, none, $\left.100,10 \mathrm{~km}\right)$ remain the same regardless of the performance levels of other sites in the portfolio. 
Assumption 4. Each set of attributes measuring the performance of a single project $X_{j I}=X_{j 1} \times$ $\cdots \times X_{j n}, j \in J$, is $W D I$.

Assumptions 1-4 are necessary for the additive-linear portfolio value function as stated by the following theorem, which uses the following notation: $x_{J i}=\left(x_{1 i}, \ldots, x_{m i}\right)^{T} \in X_{J i}$ is the $i$ th column of matrix $x \in X$, matrix $\left(x_{j i}, x_{j i}^{0}\right) \in X$ has all elements at the least preferred levels except for $x_{j i}$ and $\left(x_{J i}, x_{J \bar{i}}^{0}\right) \in X$ has all elements at the least preferred levels except for the $i$ th column $x_{J i}$.

Theorem 1. Preferences satisfy Assumptions 1-4 if and only if $V$ is either

$$
\begin{array}{ll}
\text { additive-linear: } & V(x)=\sum_{i=1}^{n} V_{i}\left(x_{J i}\right), V_{i}\left(x_{J i}\right)=w_{i}(1) \sum_{j=1}^{m} v_{i}\left(x_{j i}\right), \text { or } \\
\text { multiplicative: } & \left.1+\kappa V(x)=\prod_{i=1}^{n}\left[1+\kappa V_{i}\left(x_{J i}\right)\right], 1+\kappa V_{i}\left(x_{J i}\right)=\prod_{j=1}^{m}\left(1+\kappa w_{i}(1) v_{i}\left(x_{j i}\right)\right)\right]
\end{array}
$$

where $V_{i}: X_{J i} \rightarrow[0,1]$ and $v_{i}: X_{i} \rightarrow[0,1]$ are the criterion specific portfolio and project value functions, respectively, such that $V_{i}\left(x_{J i}\right)=V\left(x_{J i}, x_{J \bar{i}}^{0}\right)$ and $v_{i}\left(x_{j i}\right)=V\left(x_{j i}, x_{\overline{j i}}^{0}\right) / V\left(x_{j i}^{*}, x_{\overline{j i}}^{0}\right)$. Furthermore, $w_{i}(1)$ is the weight of criterion $i$ such that $w_{i}(1)=V\left(x_{j i}^{*}, x_{\overline{j i}}^{0}\right)$.

All proofs are in Appendix C.

The choice between a multiplicative and an additive-linear $V$ can be determined by examining preferences over two criteria of a single project (cf. Dyer and Sarin 1979, Corollary 1). If a change in the criterion specific performance of a project remains equally preferred when the project's performance w.r.t. some other criterion is altered, then $V$ is additive-linear. For instance, if Assumptions 1-4 hold in the site selection example then $V$ is additive-linear if and only if

$$
\left(5 \mathrm{ha}, 0 \mathrm{~m}^{3}\right) \leftarrow\left(0.5 \mathrm{ha}, 0 \mathrm{~m}^{3}\right) \sim_{D}\left(5 \mathrm{ha}, 100 \mathrm{~m}^{3}\right) \leftarrow\left(0.5 \mathrm{~h} a, 100 \mathrm{~m}^{3}\right)
$$

i.e., the preference for an increase in sites area from 0.5 to 5 ha is not dependent on the volume of old broad-leaved trees on the site. An alternative approach to establish that $V$ is additive-linear is to verify that each $X_{j I}, j \in J$, is strictly difference independent instead of Assumption 4 (see Golabi et al. 1981). 
The criterion weights $w_{1}(1), \ldots, w_{n}(1)$ can be elicited through standard tradeoff techniques. Select two criteria $i, i^{\prime} \in I$ and let $x=\left(x_{j i}^{0}, x_{j i^{\prime}}^{0}, x_{K}\right)$ be a portfolio in which project $j$ has the least preferred performance levels w.r.t. criteria $i$ and $i^{\prime}$, while all other performance levels $x_{K}$, $K=\overline{\left\{(j, i),\left(j, i^{\prime}\right)\right\}}$, are held fixed at arbitrary levels. Without loss of generality we assume that changing $x_{j i}^{0}$ to the most preferred performance level $x_{j i}^{*}$ is preferred to changing $x_{j i^{\prime}}^{0}$ to $x_{j i^{\prime}}^{*}$. The DM is asked to compensate the change $x_{j i^{\prime}}^{*} \leftarrow x_{j i^{\prime}}^{0}$ in criterion $i^{\prime}$ with a change in criterion $i$ by defining a level $x_{j i} \in X_{i}$ such that $\left(x_{j i}, x_{j i^{\prime}}^{0}, x_{K}\right) \leftarrow x \sim_{D}\left(x_{j i}^{0}, x_{j i^{\prime}}^{*}, x_{K}\right) \leftarrow x$, which implies

$$
V\left(x_{j i}, x_{j i^{\prime}}^{0}, x_{K}\right)=V\left(x_{j i}^{0}, x_{j i^{\prime}}^{*}, x_{K}\right) \Leftrightarrow v_{i}\left(x_{j i}\right) w_{i}(1)=w_{i^{\prime}}(1)
$$

since all other terms in the sum (1) or product (2) cancel out. In the site selection example this would correspond to defining site's area $y$ such that $\left(y \mathrm{ha}, 0 \mathrm{~m}^{3}\right) \sim\left(0.5 \mathrm{ha}, 200 \mathrm{~m}^{3}\right)$ when $i=1$ and $i^{\prime}=2$. Establishing equality (4) for pairs of criteria $i=1, . ., n-1, i^{\prime}=i+1$ results in $n-1$ equations that together with the chosen scaling $V\left(x^{*}\right)=1$ define a unique additive-linear $V$. The additional equation needed to define $\kappa$ in the multiplicative $V$ can be obtained by asking the DM to specify levels $x_{j i} \succ x_{i}^{0}$ and $x_{j i^{\prime}} \succ x_{i^{\prime}}^{0}$ such that $\left(x_{j i}, x_{j i^{\prime}}, x_{K}\right) \sim\left(x_{j i}^{0}, x_{j i^{\prime}}^{*}, x_{K}\right)$ (e.g., $y \in(0.5,5)$ and $y^{\prime} \in(0,200)$ such that $\left(y\right.$ ha, $\left.\left.y^{\prime} \mathrm{m}^{3}\right) \sim\left(0.5 \mathrm{ha}, 200 \mathrm{~m}^{3}\right)\right)$, which implies $\left[1+\kappa w_{i}(1) v_{i}\left(x_{j i}\right)\right]\left[1+\kappa w_{i_{i}}(1) v_{i^{\prime}}\left(x_{j i^{\prime}}\right)\right]=$ $1+\kappa w_{i^{\prime}}(1)$. These equalities clearly hold for any level of $x_{K}$, wherefore the criterion weights cannot depend on the criterion specific portfolio performance.

\section{Non-additive Portfolio Value Functions}

Due to Assumption 4 the value of adding a project into a portfolio cannot depend on how it complements the portfolio's criterion specific performances. Hence, we replace it with a weaker assumption that the attributes measuring the performance of a single project $X_{j I}$ are mutually WDI without restricting preference dependencies between projects. More specifically, we assume that if the performances of projects other than $j$ are fixed to arbitrary levels $x_{\bar{j} I} \in X_{\bar{j} I}=\times_{j^{\prime} \neq j, i \in I} X_{j^{\prime} i}$, then preferences $\succeq$ and $\succeq_{D}$ over the set of attributes $X_{j I^{\prime}}=X_{i \in I^{\prime}} X_{j i}, I^{\prime} \subset I$, do not depend on the level of the remaining attributes $X_{j \overline{I^{\prime}}}=\times_{i \notin I^{\prime}} X_{j i}$ (i.e., $X_{j I^{\prime}}$ is conditionally WDI of $X_{j \overline{I^{\prime}}}$; see Appendix A for details). 
Assumption 5. Each subset of attributes measuring the performance of a single project $X_{j I^{\prime}}, j \in$ $J, I^{\prime} \subset I$, is conditionally WDI of $X_{j \overline{I^{\prime}}}$ given a fixed level of the other attributes $X_{\bar{j} I}$.

For instance, in the site selection example it may be that when a site portfolio is empty $\left(x^{0}\right)$, criterion 'Natural water economy' $(i=3)$ is more important than 'Endangered species' $(i=4)$ in the sense that the DM would rather select a site with performances $\left(0.5 \mathrm{ha}, 0 \mathrm{~m}^{3}\right.$, exc., $\left.0,10 \mathrm{~km}\right)$ than a site with performances $\left(0.5 \mathrm{ha}, 0 \mathrm{~m}^{3}\right.$, none, $\left.100,10 \mathrm{~km}\right)$. Assumption 5 then implies $\left(y_{1} \mathrm{ha}, y_{2} \mathrm{~m}^{3}\right.$, exc., $\left.0, y_{5} \mathrm{~km}\right) \succ\left(y_{1} \mathrm{ha}, y_{2} \mathrm{~m}^{3}\right.$, none, $\left.100, y_{5} \mathrm{~km}\right)$ for any $\left(y_{1}, y_{2}, y_{5}\right) \in$ $X_{1} \times X_{2} \times X_{5}$ as long as the portfolio does not include any other sites. However, it does not rule out the possibility that $\left(y_{1}\right.$ ha, $y_{2} \mathrm{~m}^{3}$, exc., $\left.0, y_{5} \mathrm{~km}\right) \prec\left(y_{1}\right.$ ha, $y_{2} \mathrm{~m}^{3}$, none, $\left.100, y_{5} \mathrm{~km}\right)$ for all $\left(y_{1}, y_{2}, y_{5}\right) \in$ $X_{1} \times X_{2} \times X_{5}$, if the portfolio contains many sites with an excellent natural water economy, for instance.

The following theorem shows that Assumption 5 still retains the additive or multiplicative aggregation of the criterion specific portfolio value functions $V_{i}$, wherefore the same test (cf. (3)) can be used to select between these alternative forms. However, each $V_{i}$ is a symmetric strictly increasing multilinear function of the criterion specific project values $v_{i}\left(x_{1 i}\right), \ldots, v_{i}\left(x_{m i}\right)$.

TheOREm 2. Preferences satisfy Assumptions 1-3 and 5 if and only if $V$ is either

$$
\begin{aligned}
\text { additive-multilinear: } & V(x)=\sum_{i=1}^{n} V_{i}\left(x_{J i}\right), \text { or } \\
\text { multiplicative-multilinear: } & 1+\kappa V(x)=\prod_{i=1}^{n}\left[1+\kappa V_{i}\left(x_{J i}\right)\right],
\end{aligned}
$$

where the criterion specific portfolio value functions are (symmetric) multilinear:

$$
V_{i}\left(x_{J i}\right)=\sum_{J^{\prime} \subseteq J} w_{i}\left(\left|J^{\prime}\right|\right) \prod_{j \in J^{\prime}} v_{i}\left(x_{j i}\right) \prod_{j \notin J^{\prime}}\left(1-v_{i}\left(x_{j i}\right)\right) .
$$

In Equation (7) $w_{i}:\{0, \ldots, m\} \rightarrow[0,1]$ denotes the strictly increasing weighting function such that $w_{i}(k)=V_{i}\left(x_{J^{\prime} i}^{*}, x_{J^{\prime} i}^{0}\right) \forall J^{\prime} \subseteq J,\left|J^{\prime}\right|=k$ and $w_{i}(0)=V_{i}\left(x_{J i}^{0}\right)=0$.

In the value functions of Theorem 1 the 'importance' of each criterion is captured by a single parameter $w_{i}(1)$, but additive- and multiplicative-multilinear $V$ deploy $m$ parameters 
$w_{i}(1), \ldots, w_{i}(m)$. Each $w_{i}(k)$ corresponds to the criterion specific value of a portfolio that has $k$ projects with indexes $J^{\prime} \subseteq J$ on the most preferred level $x_{i}^{*}$ and the other $m-k$ projects on the least preferred level $x_{i}^{0}$ (denoted by $\left(x_{J^{\prime} i}, x_{\overline{J^{\prime}} i}\right)$ ). Due to project-symmetric preferences (Assumption 1) $V_{i}\left(x_{J^{\prime} i}, x_{\overline{J^{\prime}} i}\right)$ remains constant for any $J^{\prime}$ whose size is $k$. To make the notation more compact we denote $\left\langle k, x_{i}^{*}\right\rangle=\left(x_{1 i}^{*}, \ldots, x_{k i}^{*}, x_{i}^{0}, \ldots, x_{i}^{0}\right)^{T}$ and $w_{i}(k)=V_{i}\left(\left\langle k, x_{i}^{*}\right\rangle\right)$. More generally, we use $\left\langle k_{1}, y ; k_{2}, y^{\prime}\right\rangle \in X_{J i}$ to denote the criterion specific performance of a portfolio with $k_{1}$ projects at performance level $y \in X_{i}, k_{2}$ projects at performance level $y^{\prime} \in X_{i}$, and the rest of the projects at the least preferred level $x_{i}^{0}$, i.e.,

$$
\left\langle k_{1}, y ; k_{2}, y^{\prime}\right\rangle=(\underbrace{y, \ldots, y}_{\begin{array}{c}
k_{1} \\
\text { elements }
\end{array}}, \underbrace{y^{\prime}, \ldots, y^{\prime}}_{\begin{array}{c}
k_{2} \\
\text { elements }
\end{array}}, \underbrace{x_{i}^{0}, \ldots, x_{i}^{0}}_{\begin{array}{c}
m-k_{1}-k_{2} \\
\text { elements }
\end{array}})^{T} \in X_{J i} .
$$

\subsection{Eliciting Criterion Specific Portfolio Value Functions}

When the criterion specific project value function $v_{i}$ has been established, eliciting $V_{i}(7)$ requires defining the weighting function values $w_{i}(1), \ldots, w_{i}(m)$. This can be done by first fixing the other criteria to any level $x_{J \bar{i}}$ and then building equally preferred portfolios $(\sim)$ or equally preferred changes between portfolios $\left(\sim_{d}\right)$ that only differ w.r.t. criterion $i$.

One approach is to go through the values $k \in\{1, \ldots, m-1\}$ and for each $k$ ask the DM to adjust the level $y \in X_{i}$ until the portfolios $\left\langle k, x_{i}^{*}\right\rangle$ and $\left\langle k-1, x_{i}^{*} ; 2, y\right\rangle$ are equally preferred. For instance, applying this approach for criterion 'Old broad-leaved trees' with $k=10$ requires the DM to define a volume $y$ between 0 and $200 \mathrm{~m}^{3}$ such that having nine sites with $200 \mathrm{~m}^{3}$ and two sites with $y \mathrm{~m}^{3}$ of broad-leaved trees is equally preferred to having ten sites with $200 \mathrm{~m}^{3}$ of broad-leaved trees. In terms of criterion specific portfolio value $\left\langle k, x_{i}^{*}\right\rangle \sim\left\langle k-1, x_{i}^{*} ; 2, y\right\rangle$ is equivalent to

$$
\begin{aligned}
V_{i}\left(\left\langle k, x_{i}^{*}\right\rangle\right) & =V_{i}\left(\left\langle k-1, x_{i}^{*} ; 2, y\right\rangle\right) \\
\Leftrightarrow w_{i}(k) & =v_{i}^{2}(y) w_{i}(k+1)+2 v_{i}(y)\left(1-v_{i}(y)\right) w_{i}(k)+\left(1-v_{i}(y)\right)^{2} w_{i}(k-1) \\
\Leftrightarrow w_{i}(k+1)-w_{i}(k) & =\left(\frac{1-v_{i}(y)}{v_{i}(y)}\right)^{2}\left(w_{i}(k)-w_{i}(k-1)\right) .
\end{aligned}
$$

Notice that if $y=x_{i}^{0}\left(\right.$ or $\left.y=x_{i}^{*}\right)$ then $\left\langle k, x_{i}^{*}\right\rangle \sim\left\langle k-1, x_{i}^{*}\right\rangle\left(\right.$ or $\left.\left\langle k, x_{i}^{*}\right\rangle \sim\left\langle k+1, x_{i}^{*}\right\rangle\right)$, which contradicts the requirement that $w_{i}$ is strictly increasing. Since $v_{i}(y)$ is a known numerical value in the interval 
$(0,1)$, Equation (9) is a linear constraint on the weighting function values (in fact comparison of any two performances or changes in $X_{J i}$ results in a linear constraint; see Appendix B for details). Repeating this question for each $k=1, \ldots, m-1$ results in $m-1$ linear equations for $m$ variables which determines the values of $w_{i}(1), \ldots, w_{i}(m)$ up to multiplication with a positive constant.

Another approach is to apply standard value function elicitation techniques (see, e.g., von Winterfeldt and Edwards 1986) over the discrete measurement scale $\{0,1,2, \ldots, m\}$ to obtain the criterion specific values of portfolios consisting of $k$ and $m-k$ projects with performance levels $x_{i}^{*}$ and $x_{i}^{0}$, respectively. For instance, applying the difference standard sequence to the criterion 'Natural water economy' $(i=3)$ starts by defining a unit stimulus, say the change from a portfolio with no water economy $\left(x_{J 3}^{0}\right)$ to a portfolio of 10 sites with excellent water economy, i.e., $\left\langle k_{0}\right.$, exc. $\rangle$, where $k_{0}=10$ (see Figure 3). Then the DM is asked to define the number of projects $k_{1}$, such that the change from $k_{0}=10$ to $k_{1}$ sites with excellent natural water economy is equally preferred to the change from zero to $k_{0}=10$ such sites $\left(k_{1}=13\right.$ in Figure 3$)$. Continuing this process results in a sequence of portfolio performances $\left\langle k_{1}\right.$, exc. $\rangle,\left\langle k_{2}\right.$, exc. $\rangle$, etc., where each change $\left\langle k_{l}\right.$, exc. $\rangle \leftarrow\left\langle k_{l-1}\right.$, exc. $\rangle$ is equally preferred. The equality $V_{3}\left(\left\langle k_{l}, x_{i}^{*}\right\rangle\right)-V_{3}\left(\left\langle k_{l-1}, x_{i}^{*}\right\rangle\right)=w_{3}\left(k_{l}\right)-w_{3}\left(k_{l-1}\right)=w_{3}\left(k_{0}\right)-w_{3}(0)=w_{3}\left(k_{0}\right)$ then defines the weighting function value for each $k_{l}$ and the rest of the values can be obtained by linear interpolation or by applying technique (8). Again the weighting function values are determined up to multiplication with a positive constant since the value of the unit stimulus $w_{3}\left(k_{0}\right)$ remains free.

\subsection{Aggregating the Criterion Specific Portfolio Values}

To fix the absolute weighting function values requires defining the tradeoffs among the criteria. In addition to the scaling constraint $V\left(x^{*}\right)=1, n-1$ or $n$ constraints are needed to establish a unique additive- or multiplicative-multilinear $V$, respectively. These constraints can be obtained with similar techniques as with the additive-linear and multiplicative $V$ : Let $x=\left(x_{j i}^{0}, x_{j i^{\prime}}^{0}, x_{K}\right)$ be a portfolio in which project $j$ has the least preferred performance levels w.r.t. criteria $i$ and $i^{\prime}$, while all other performance levels $x_{K}$ are held fixed at arbitrary levels. The DM is asked to compensate 


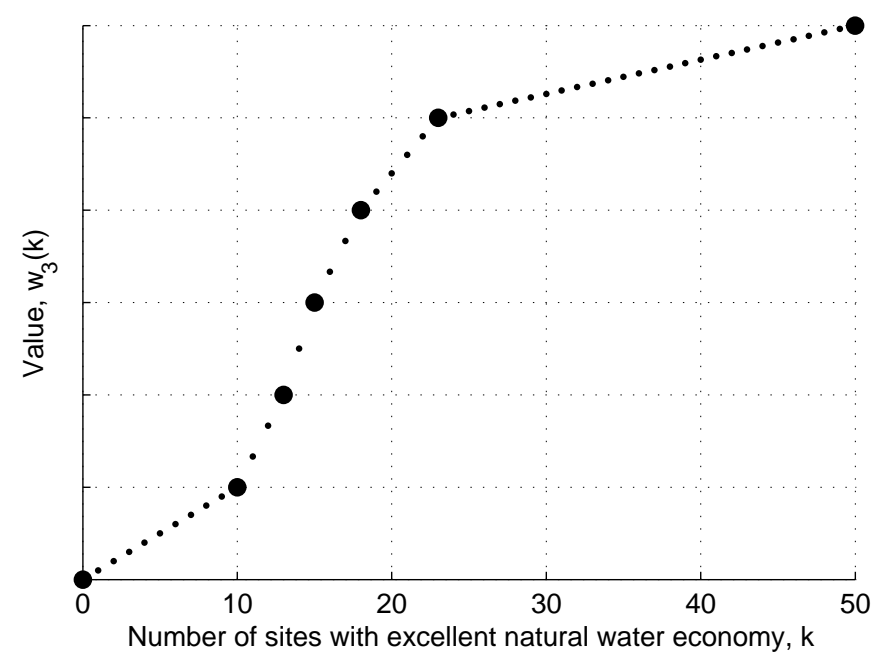

Figure 3 Large dots correspond to the weighting function values obtained from the difference standard sequence $k_{l}=0,10,13,15,18,23,50$ and small dots to the interpolated values.

the change $x_{j i^{\prime}}^{*} \leftarrow x_{j i^{\prime}}^{0}$ in criterion $i^{\prime}$ with a change in criterion $i$ by defining a level $x_{j i} \in X_{i}$ such that

$$
\begin{gathered}
\left(x_{j i}, x_{j i^{\prime}}^{0}, x_{K}\right) \leftarrow\left(x_{j i}^{0}, x_{j i^{\prime}}^{0}, x_{K}\right) \sim_{D}\left(x_{j i}^{0}, x_{j i^{\prime}}^{*}, x_{K}\right) \leftarrow\left(x_{j i}^{0}, x_{j i^{\prime}}^{0}, x_{K}\right) \\
\Leftrightarrow V\left(x_{j i}, x_{j i^{\prime}}^{0}, x_{K}\right)=V\left(x_{j i}^{0}, x_{j i^{\prime}}^{*}, x_{K}\right) .
\end{gathered}
$$

If the DM compares the criterion specific performances of a single project when the rest of the projects are on the least preferred level, i.e., $x_{K}=x_{K}^{0}$, then (10) implies $v_{i}\left(x_{j i}\right) w_{i}(1)=w_{i^{\prime}}(1)$, which is equivalent to Equation (4) obtained for the multiplicative/additive-linear $V$.

The DM can also be asked to define a corresponding compensation when the portfolio already contains some projects and hence $x_{K} \neq x_{K}^{0}$. In the site selection example, for instance, the DM could be asked to first consider a portfolio with $k=25$ sites, each with $200 \mathrm{~m}^{3}$ of old broad-leaved trees $(i=2)$ and an excellent natural water economy $\left(i^{\prime}=3\right)$. Then she would define $y \in X_{2}$ such that adding either of the sites $\left(\cdot, y \mathrm{~m}^{3}\right.$, none, $\left., \cdot, \cdot\right)$ and $\left(\cdot, 0 \mathrm{~m}^{3}\right.$, exc., $\left.\cdot, \cdot\right)$ into the portfolio would be equally preferred, i.e.,

$$
\left(\left\langle 25,200 \mathrm{~m}^{3} ; 1, y \mathrm{~m}^{3}\right\rangle,\langle 25, \text { exc. }\rangle\right) \leftarrow\left(\left\langle 25,200 \mathrm{~m}^{3}\right\rangle,\langle 25, \text { exc. }\rangle\right) \sim_{D}
$$




$$
\left(\left\langle 25,200 \mathrm{~m}^{3}\right\rangle,\langle 26, \text { exc. }\rangle\right) \leftarrow\left(\left\langle 25,200 \mathrm{~m}^{3}\right\rangle,\langle 25, \text { exc. }\rangle\right) .
$$

In general, if $x_{K}$ has $k$ projects on the most preferred performance level w.r.t criterion $i$ and $k^{\prime}$ projects on the most preferred performance level w.r.t criterion $i^{\prime}$, then Equation (10) yields

$$
\begin{aligned}
v_{i}\left(x_{j i}\right)\left[w_{i}(k+1)-w_{i}\left(k_{i}\right)\right] & =w_{i^{\prime}}\left(k^{\prime}+1\right)-w_{i^{\prime}}\left(k^{\prime}\right), \\
\frac{1+\kappa\left[v_{i}\left(x_{j i}\right) w_{i}(k+1)+\left(1-v_{i}\left(x_{j i}\right)\right) w_{i}(k)\right]}{1+\kappa w_{i}(k)} & =\frac{1+\kappa w_{i^{\prime}}\left(k^{\prime}+1\right)}{1+\kappa w_{i^{\prime}}\left(k^{\prime}\right)}
\end{aligned}
$$

for the additive- and multiplicative-multilinear $V$, respectively. Repeating this question for the pairs of criteria $i=1, \ldots, n-1, i^{\prime}=i+1$ results in $n-1$ constraints and the additional constraint needed for the multiplicative-multilinear $V$ can be elicited similarly as with the multiplicative $V$.

When $V$ is additive-multilinear standard weighting techniques can be applied to assess the tradeoffs among the criteria. For instance, in swing weighting (von Winterfeldt and Edwards 1986) the DM first determines the order in which she would prefer to change the criteria from the least to the most preferred level and then assesses swing weights $s_{1}, \ldots, s_{n}$ that reflect the value increases of these changes. In our setting these changes correspond to $x_{J i}^{*} \leftarrow x_{J i}^{0}, i \in I$, and hence the normalized swing weights are equal to $w_{1}(m), \ldots, w_{n}(m)$. In turn, asking the DM to consider changes $\left\langle k, x_{i}^{*}\right\rangle \leftarrow x_{J i}^{0}$, $i \in I$, results in swing weights such that $s_{i} / s_{i^{\prime}}=w_{i}(k) / w_{i^{\prime}}(k)$ for all $i, i^{\prime} \in I$.

\subsection{Special Forms of the Multilinear Criterion Specific Portfolio Value Function}

The techniques for eliciting $V_{i}$ in Section 4.1 produce linear constraints for the weighting function values $w_{i}(1), \ldots, w_{i}(m)$. If these constraints imply that the ratio $r_{i}$ between successive value increases $w_{i}(k+1)-w_{i}(k)$ and $w_{i}(k)-w_{i}(k-1)$ is constant for any $k \in\{1, \ldots, m-1\}$, then $V_{i}$ is either multiplicative $\left(r_{i} \neq 1\right)$ or linear $\left(r_{i}=1\right)$. For instance, in the site selection example the DM may feel that a site with excellent natural water economy is equally preferred to two sites with good natural water economy. This statement does not depend on what other sites are included in the portfolio and hence implies $\langle k$, exc. $\rangle \sim\langle k-1$, exc.; 2, good $\rangle$ for all $k \in\{1, \ldots, m-1\}$. Using Equation (9) this can be written as $w_{3}(k+1)-w_{3}(k)=r_{3}\left[w_{3}(k)-w_{3}(k-1)\right]$, where $r_{3}=\left[1 / v_{3}(\operatorname{good})-1\right]^{2}=0.44$. 
COROLlary 1. Let $V_{i}$ be symmetric multilinear (7). There exists $r_{i} \in(0, \infty)$ such that the weighting function $w_{i}$ satisfies $w_{i}(k+1)-w_{i}(k)=r_{i}\left[w_{i}(k)-w_{i}(k-1)\right]$ for all $k \in\{1, \ldots, m-1\}$ if and only if the criterion specific portfolio value function is either

$$
\begin{aligned}
\text { linear: } \quad V_{i}\left(x_{J i}\right) & =w_{i}(1) \sum_{j=1}^{m} v_{i}\left(x_{j i}\right) \text { or } \\
\text { multiplicative: } \quad V_{i}\left(x_{J i}\right) & =\frac{w_{i}(1)}{r_{i}-1}\left[\prod_{j=1}^{m}\left(1+\left(r_{i}-1\right) v_{i}\left(x_{j i}\right)\right)-1\right] \text { for some } r_{i} \in(0, \infty) \backslash\{1\} .
\end{aligned}
$$

In some cases the sum of the projects' criterion specific performances $\sum_{j=1}^{m} x_{j i}$ has a natural interpretation as a measure of portfolio performance (cf. volume of old broad-leaved trees in the site selection example). Corollary 2 shows that there are two special forms of symmetric multilinear $V_{i}$ that represent preferences over the sum of the projects' criterion specific performances. The first of these forms is obtained by substituting the linear criterion specific project value function $v_{i}\left(x_{j i}\right)=\left(x_{j i}-x_{i}^{0}\right) /\left(x_{i}^{*}-x_{i}^{0}\right)$ into the linear criterion specific portfolio value function $V_{i}(13)$ which gives

$$
V_{i}\left(x_{J i}\right)=w_{i}(1) \frac{\sum_{j=1}^{m} x_{j i}-m x_{i}^{0}}{x_{i}^{*}-x_{i}^{0}}
$$

In turn, substituting the exponential $v_{i}\left(x_{j i}\right)=\left[1-e^{-\left(x_{j i}-x_{i}^{0}\right) / \rho}\right] /\left[1-e^{-\left(x_{i}^{*}-x_{i}^{0}\right) / \rho}\right]$, where $\rho \in \mathbb{R} \backslash\{0\}$, into the multiplicative $V_{i}$ (14) with $r_{i}=e^{-\left(x_{i}^{*}-x_{i}^{0}\right) / \rho}$ results in the exponential value function

$$
V_{i}\left(x_{J i}\right)=w_{i}(1) \frac{1-e^{-\left(\sum_{j=1}^{m} x_{j i}-m x_{i}^{0}\right) / \rho}}{1-e^{-\left(x_{i}^{*}-x_{i}^{0}\right) / \rho}} .
$$

Corollary 2. Let $V_{i}$ be symmetric multilinear (7). $V_{i}$ represents preferences $\left(x_{J i}^{a}, x_{J \bar{i}}\right) \succeq$ $\left(x_{J i}^{b}, x_{J \bar{i}}\right) \Leftrightarrow \sum_{j=1}^{m} x_{j i}^{a} \geq \sum_{j=1}^{m} x_{j i}^{b}$ if and only if $V_{i}$ is equal to either (15) or (16).

\subsection{Comparison of Multilinear and Linear Criterion Specific Portfolio Value Functions}

Figure 4 illustrates how the developed multilinear $V_{i}$ compares to the standard linear $V_{i}(13)$, i.e., the sum of the projects' criterion specific values (scores). Each of Figures $4 a-d$ shows a weighting function $w_{i}(0), \ldots, w_{i}(m)(m=10,50)$ marked with black dots and a projection of the resulting multilinear $V_{i}$ : the gray area is the set of points $\left(\sum_{j=1}^{m} v_{i}\left(x_{j i}\right), V_{i}\left(x_{J i}\right)\right)$ obtained when the portfolio 
performance $x_{J i}=\left(x_{1 i}, \ldots, x_{m i}\right)^{T}$ is varied through its entire domain $X_{J i}=X_{i} \times \ldots \times X_{i}$. $\left(v_{i}\right.$ is assumed continuous and hence each $v_{i}\left(x_{j i}\right)$ obtains all values in the interval $[0,1]$, see Appendix $\mathrm{B}$ for details). The black dots are inside the gray area since $\left(\sum_{j=1}^{m} v_{i}\left(x_{j i}\right), V_{i}\left(x_{J i}\right)\right)=\left(k, w_{i}(k)\right)$ whenever $x_{J i}=\left\langle k, x_{i}^{*}\right\rangle$.
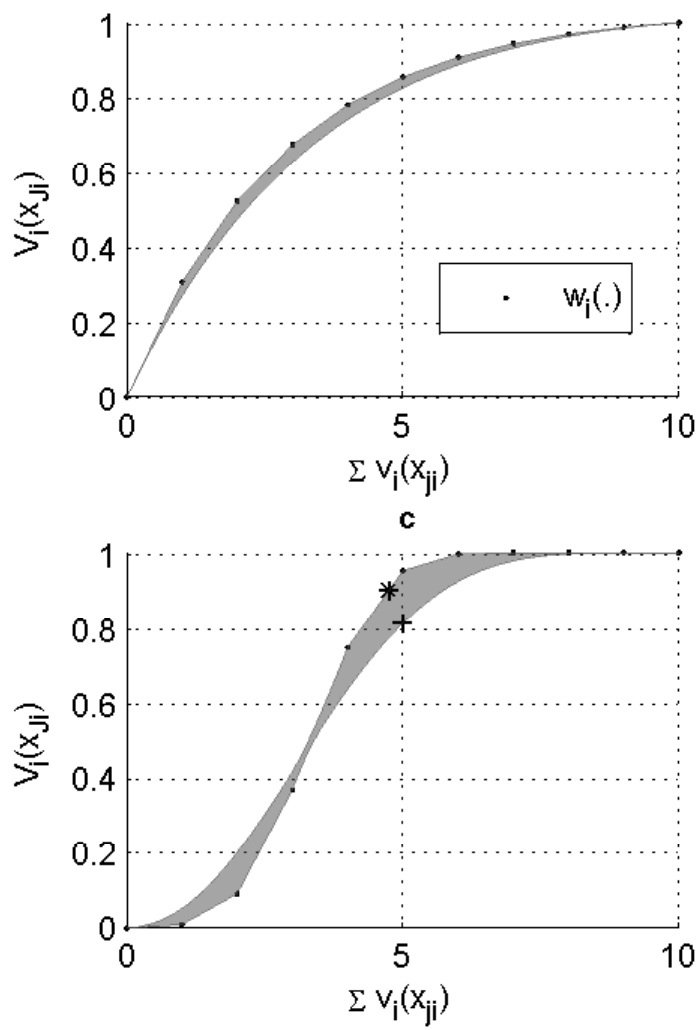

b
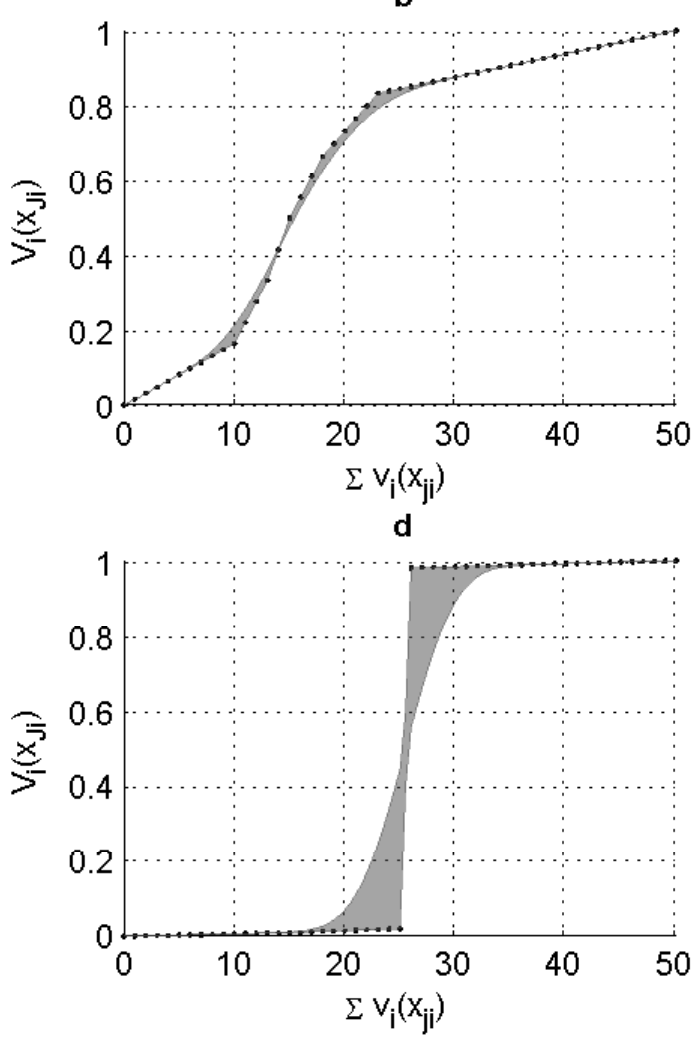

Figure 4 Multilinear $V_{i}$ and the sum of criterion specific project values for four weighting functions $w_{i}$ : (a) $w_{i}(k+1)-w_{i}(k)=0.7\left(w_{i}(k)-w_{i}(k-1)\right.$ for all $k=1, \ldots, 9($ cf. Corollary 1$),\left(\right.$ b) $w_{i}$ is obtained by the difference standard technique (cf. Figure 3), (c) $w_{i}$ follows the cumulative normal distribution function, and (d) $w_{i}$ is roughly equal to a step function.

Figure 4c illustrates that $V_{i}$ is not necessarily increasing in the sum of scores; For instance, portfolios $\langle 10, y\rangle, v_{i}(y)=0.5$ (marked with '+'), and $\left\langle 5, x_{i}^{*}\right\rangle$ have criterion specific values 0.81 and $0.95=w_{i}(5)$, respectively, even though the sum of the projects' scores for both portfolios is 5 . Furthermore, portfolio $\left\langle 4, x^{*} ; 1, y^{\prime}\right\rangle$, where $v_{i}\left(y^{\prime}\right)=0.75$ (marked with ${ }^{*}{ }^{\prime}$ ) has a criterion specific 
value of 0.89 even though the sum of its projects' scores is only 4.75 . This highlights that even though the criterion specific project value functions $v_{i}$ are measurable (i.e. cardinal), the sum of project scores $\sum_{j=1}^{m} v_{i}\left(x_{j i}\right)$ does not provide a portfolio performance measure in the sense that preferences are representable with a non-linear value function over this sum. Yet, it is somewhat surprising that in some cases such an approach is almost equal to applying a multilinear $V_{i}$. For instance, in Figure 4a the maximum criterion specific value difference of two portfolios with equal sum of scores is only 0.05 . In turn, the other extreme is illustrated in Figure $4 \mathrm{~d}$ where $w_{i}$ is roughly equal to a step function and the maximum value difference is close to 0.5 .

\subsection{The Multilinear Portfolio Value Function}

We conclude this section by briefly discussing the case where preferences do not satisfy neither of Assumptions 4 and 5.

Theorem 3. Preferences satisfy Assumptions 1-3 if and only if $V$ is multilinear:

$$
V(x)=\sum_{I^{\prime} \subseteq I} \lambda\left(I^{\prime}\right) \prod_{i \in I^{\prime}} \frac{V_{i}\left(x_{J i}\right)}{\lambda(\{i\})} \prod_{i \notin I^{\prime}}\left(1-\frac{V_{i}\left(x_{J i}\right)}{\lambda(\{i\})}\right),
$$

where $\lambda\left(I^{\prime}\right)=V\left(x_{J I^{\prime}}^{*}, x_{\overline{J I^{\prime}}}^{0}\right), I^{\prime \prime} \subset I^{\prime} \Rightarrow \lambda\left(I^{\prime \prime}\right)<\lambda\left(I^{\prime}\right), V\left(x^{0}\right)=\lambda(\emptyset)=0, V\left(x^{*}\right)=\lambda(I)=1$ and the criterion specific portfolio value functions $V_{i}$ are symmetric multilinear (7). Furthermore, (5) and (6) are obtained from (17) by substituting $\lambda\left(I^{\prime}\right)=\sum_{i \in I^{\prime}} \lambda(\{i\})$ and $1+\kappa \lambda\left(I^{\prime}\right)=\prod_{i \in I^{\prime}}(1+\kappa \lambda(\{i\}))$, respectively.

In the multilinear $V$ each $\lambda$ corresponds to the value of a portfolio with each criterion $i$ either at the most preferred level $x_{J i}^{*}$ or at the least preferred level $x_{J i}^{0}$. For instance, in the site selection example $\lambda(\{1,2\})=V(x)$, where portfolio $x$ consists of $m$ projects with performances $\left(x_{1}^{*}, x_{2}^{*}, x_{3}^{0}, x_{4}^{0}, x_{5}^{0}\right)=$ $\left(5 \mathrm{ha}, 200 \mathrm{~m}^{3}\right.$, none, $\left.0,50 \mathrm{~km}\right) . V_{i}$ can be elicited with the techniques presented in Section 4.1, after which the values of $\lambda$ can be obtained by examining preferences over the performances of a single project $X_{1} \times \cdots \times X_{n}$. More specifically, by setting $x_{j^{\prime} i}=x_{i}^{0}$ for all $j^{\prime} \in J \backslash\{j\}, i \in I$, (17) becomes

$$
V(x)=\sum_{I^{\prime} \subseteq I} \lambda\left(I^{\prime}\right) \prod_{i \in I^{\prime}} v_{i}\left(x_{j i}\right) \frac{w_{i}(1)}{w_{i}(m)} \prod_{i \notin I^{\prime}}\left(1-v_{i}\left(x_{j i}\right) \frac{w_{i}(1)}{w_{i}(m)}\right),
$$


where $w_{i}(1) / w_{i}(m)$ and $v_{i}\left(x_{j i}\right)$ are known numbers. However, the elicitation burden grows exponentially in the number of criteria $n$, wherefore in most cases it may be more practical to reformulate the evaluation criteria (cf. Keeney and von Winterfeldt 2007) so that Assumption 5 holds and thus less complex additive- or multiplicative-multilinear portfolio value functions of Theorem 2 can be used.

\section{Optimization Models for Maximizing Portfolio Value}

The most preferred portfolio that satisfies the feasibility constraints can be identified by solving the integer programming problem

$$
\max _{z \in Z_{F}} V(\hat{x}(z)), Z_{F}=\left\{z \in\{0,1\}^{m} \mid A z \leq B\right\}
$$

where function $\hat{x}:\{0,1\}^{m} \rightarrow X$ maps the vector of decision variables $z$ to performance levels $\hat{x}(z)$ such that for each project $j, \hat{x}_{j I}(z)=\left(x_{j 1}^{g o}, \ldots, x_{j n}^{g o}\right)$ if $z_{j}=1$ and $\hat{x}_{j I}(z)=\left(x_{j 1}^{n o}, \ldots, x_{j n}^{n o}\right)$ if $z_{j}=0$. To our knowledge there exist no well established algorithms to solve (18) when $V$ is not additive-linear (1). Hence, we develop two approaches for solving (18): An exact implicit enumeration algorithm for small problems $(m<100)$ and an approximate mixed integer linear programming (MILP) model for large problems $(m>100)$ with an additive-multilinear $V(5)$.

\subsection{Implicit Enumeration Algorithm}

To keep the notation simple we first introduce the implicit enumeration algorithm with a single feasibility constraint $a z \leq b$ and then extend it to handle multiple constraints. The algorithm enumerates the possible values of decision variables $z$ starting with $z=(0, \ldots, 0)^{T}$, and composes of two main parts: The Forward-loop and the Backtrack-step. The Forward-loop successively sets $z_{k} \leftarrow 1$ in an increasing order of indexes $k$ and at each iteration in which $z$ is feasible compares the value of portfolio $\hat{x}(z)$ to the greatest portfolio value found so far, $V^{*}$. If $V(\hat{x}(z))$ is greater than $V^{*}$, then $z$ is stored as the best solution $z^{*}$. The Forward-loop is terminated when $k$ reaches $m$, whereafter the algorithm moves to the Backtrack-step. The Backtrack-step sets $z_{m} \leftarrow 0$, finds the 
greatest index $k$ with $z_{k}=1$ and sets $z_{k} \leftarrow 0$. If such an index does not exist, then the algorithm terminates; otherwise the Forward-loop is repeated.

Two additional steps are implemented into the Forward-loop to avoid enumeration of all $2^{m}$ possible values of $z$. These steps utilize the property that at any time $z_{j}=0$ for all $j>k$, and continuing the Forward-loop can only change the values of $z_{k+1}, \ldots, z_{m}$ to one. First, if the solution $z$ is infeasible and it cannot be made feasible by setting $z_{j}=1$ for some indexes $j \in\{k, \ldots, m\}$, then there is no need to continue the Forward-loop as it would only produce infeasible solutions. Second, if the portfolio value $V(\hat{x}(z))$ that can be obtained by setting $z_{j}=1$ for some indexes $j \in\{k, \ldots, m\}$ cannot exceed $V^{*}$, then continuing the Forward-loop would only produce sub-optimal solutions. To determine whether this conditions holds, we compute an upper bound $s_{i}$ for the sum of criterion specific projects' values $\sum_{j=1}^{m} v_{i}\left(\hat{x}_{j i}(z)\right)$ when $z_{1}, \ldots, z_{k}$ are fixed and $z_{k+1}, \ldots, z_{m}$ are optimized subject to the feasibility constraint, i.e.,

$$
s_{i} \leftarrow \sum_{j=1}^{k} v_{i}\left(\hat{x}_{j i}(z)\right)+\max _{\substack{\tilde{z}_{j} \in[0,1] \\ k<j \leq m}}\left\{\sum_{j=k+1}^{m} \tilde{z}_{j} v_{i}\left(x_{j i}^{g o}\right)+\left(1-\tilde{z}_{j}\right) v_{i}\left(x_{j i}^{n o}\right) \mid \sum_{j=k+1}^{m} \tilde{z}_{j} a_{j} \leq b-\sum_{j=1}^{k} z_{j} a_{j}\right\} .
$$

In (19) the integrality constraints on $z_{k+1}, \ldots, z_{m}$ are relaxed to obtain a linear programming problem that can be solved efficiently (see, e.g., Martello and Toth 1990). The upper bound $s_{i}$ for the sum of scores implies also an upper bound $\bar{V}_{i}\left(s_{i}\right)$ for each $V_{i}\left(\hat{x}_{J i}(z)\right)$ (see Figure 4 and Appendix B for details). Furthermore, $V$ can be written as $V(x)=g\left(V_{1}\left(x_{J 1}\right), \ldots, V_{n}\left(x_{J n}\right)\right)$ where $g: \mathbb{R}^{n} \rightarrow \mathbb{R}$ is increasing in all arguments, wherefore $g\left(\bar{V}_{1}\left(s_{1}\right), \ldots, \bar{V}_{n}\left(s_{n}\right)\right)$ is an upper bound for the portfolio value $V(\hat{x}(z))$. Formally the implicit enumeration algorithm can be stated as follows:

$$
\begin{aligned}
& z \leftarrow(0, \ldots, 0)^{T}, k \leftarrow 0, z^{*} \leftarrow \emptyset, V^{*} \leftarrow-\infty \\
& \text { If } \sum_{j=1}^{m} z_{j} a_{j} \leq b \text { then } z^{*} \leftarrow z, V^{*} \leftarrow V(\hat{x}(z))
\end{aligned}
$$

Repeat

Repeat (Forward-loop)

$$
\begin{aligned}
& k \leftarrow k+1, z_{k} \leftarrow 1 \\
& \text { If } \sum_{j=1}^{m} z_{j} a_{j} \leq b \text { and } V(\hat{x}(z))>V^{*} \text { then } z^{*} \leftarrow z, V^{*} \leftarrow V(\hat{x}(z)) \\
& \text { If } \sum_{j=1}^{k} z_{j} a_{j}+\sum_{j=k+1}^{m} \min \left\{0, a_{j}\right\}>b \text { then break loop }
\end{aligned}
$$


If $g\left(\bar{V}_{1}\left(s_{1}\right), \ldots, \bar{V}_{n}\left(s_{n}\right)\right)<V^{*}$ then break loop

Until $k=m$

Backtrack-step:

$z_{m} \leftarrow 0$

$k \leftarrow \max \left(\left\{j \in J \mid z_{j}=1\right\} \cup\{0\}\right)$

If $k>0$ then $z_{k} \leftarrow 0$

Until $k=0$

THEOREM 4. Let $z^{*}$ be the output of the implicit enumeration algorithm. If $z^{*}=\emptyset$ then $\{z \in$ $\left.\{0,1\}^{m} \mid a z \leq b\right\}=\emptyset$; otherwise $z^{*} \in \arg \max _{z \in\{0,1\}^{m}}\{V(\hat{x}(z)) \mid a z \leq b\}$.

Extending the algorithm to account for $q>1$ feasibility constraints $A z \leq B$ is straightforward. First, if $\sum_{j=1}^{k} z_{j} A_{l j}+\sum_{j=k+1}^{m} \min \left\{0, A_{l j}\right\}>B_{l}$ holds for any $l=1, \ldots, q$, then the $l$ th constraint cannot be satisfied by setting $z_{j}=1$ for any $j \in\{k, \ldots, m\}$. Thus, one or more variables $z_{j}=1, j \leq k$, have to be set to zero to obtain a feasible solution and the Forward-loop can be terminated. Second, an upper bound for the solution of optimization problem (19) with $q$ feasibility constraints and $m-k$ integrality constraints can be obtained by minimizing its Lagrangean dual with subgradient optimization (see, e.g., Bertsimas and Tsitsiklis 1997).

\subsection{MILP Approximation}

If $V$ is additive-multilinear, problem (18) can be approximated with a MILP model. This requires choosing a piecewise linear mapping $\tilde{V}_{i}:[0, m] \rightarrow[0,1]$ for each criterion $i \in I$ such that the difference between the exact criterion specific portfolio value $V_{i}\left(x_{J i}\right)$ and its approximation $\tilde{V}_{i}\left(\sum_{j=1}^{m} v_{i}\left(x_{j i}\right)\right)$ is 'small' for all $x_{J i} \in X_{J i}$. Let $\chi_{1}^{i}<\chi_{2}^{i} \ldots<\chi_{l_{i}}^{i}$ denote the real-valued points on the interval $[0, m]$ such that $\tilde{V}_{i}$ is linear on each interval $\left[\chi_{d}^{i}, \chi_{d+1}^{i}\right]$. Then the problem $\max _{z \in\{0,1\}^{m}}\left\{\sum_{i=1}^{n} \tilde{V}_{i}\left(\sum_{j=1}^{m} v_{i}\left(\hat{x}_{j i}(z)\right)\right) \mid A z \leq B\right\}$ can be formulated as the following MILP problem (see, e.g., Bertsimas and Tsitsiklis 1997):

$$
\begin{array}{cl}
\max _{\substack{z \in\{0,1\}^{m} \\
\theta^{i}, \psi^{i}, i \in I}} & \sum_{i=1}^{n} \sum_{d=1}^{l_{i}} \theta_{d}^{i} \tilde{V}_{i}\left(\chi_{d}^{i}\right) \\
& A z \leq B
\end{array}
$$




$$
\begin{aligned}
& \sum_{j=1}^{n} z_{j} v_{i}\left(x_{j i}^{g o}\right)+\left(1-z_{j}\right) v_{i}\left(x_{j i}^{n o}\right)=\sum_{d=1}^{l_{i}} \theta_{d}^{i} \chi_{d}^{i} \forall i \in I \\
& \sum_{d=1}^{l_{i}} \theta_{d}^{i}=1 \forall i \in I \\
& \sum_{d=1}^{l_{i}-1} \psi_{d}^{i}=1 \forall i \in I \\
& \theta_{1}^{i} \leq \psi_{1}^{i} \forall i \in I \\
& \theta_{d}^{i} \leq \psi_{d-1}^{i}+\psi_{d}^{i} \forall d \in\left\{2, \ldots ., l_{i}-1\right\} i \in I \\
& \theta_{l_{i}}^{i} \leq \psi_{l_{i}-1}^{i} \forall i \in I \\
& \theta^{i} \in[0,1]^{l_{i}}, \psi^{i} \in\{0,1\}^{l_{i}-1}, \forall i \in I .
\end{aligned}
$$

The number of additional variables and constraints in the MILP problem (20)-(28) depends on the number of points $\chi_{d}^{i}$ used in the approximations $\tilde{V}_{i}, i=1, \ldots, n$. For instance, the $V_{i}$ illustrated in Figure 4b can be approximated by seven points $\chi^{i}=(0,10,13,15,18,23,50)$ with values $\tilde{V}_{i}\left(\chi_{d}^{i}\right)=w_{i}\left(\chi_{d}^{i}\right)$ requiring only 13 additional variables of which 6 are integer valued and 9 additional constraints $(23)-(27)$. If $\tilde{V}_{i}$ is concave there is no need to introduce the binary variables $\psi^{i}$ and the associated constraints $(24)-(27)$, because $\tilde{V}_{i}\left(\sum_{d=1}^{l_{i}} \theta_{d}^{i} \chi_{d}^{i}\right) \geq \sum_{d=1}^{l_{i}} \theta_{d}^{i} \tilde{V}_{i}\left(\chi_{d}^{i}\right)$ for any non-negative $\theta_{1}, \ldots, \theta_{l_{i}}$ that sum up to one. For instance, the $V_{i}$ illustrated in Figure 4a can be approximated by 11 points $\chi^{i}=(0,1, \ldots, 10)$ with values $\tilde{V}_{i}\left(\chi_{d}^{i}\right)=w_{i}\left(\chi_{d}^{i}\right)$ resulting in 11 additional continuous variables $\theta^{i}$ and one additional constraint (23).

\section{Conservation Site Selection Example Revisited}

We continue with the site selection example to illustrate use of the additive-multilinear portfolio value function and the developed optimization models. This requires eliciting the criterion specific portfolio value functions $V_{1}, \ldots, V_{5}$ (see Figure 5) in addition to the criterion specific site value functions $v_{1}, \ldots, v_{5}$ specified in Figure 1 . The marginal value of every additional conservation hectare is constant, wherefore both $v_{1}$ and $V_{1}$ are linear. The volume of 'Old broad-leaved trees' is a natural performance measure of both sites and site portfolios, wherefore the use of an exponential value function (16) over the net volume is appropriate when the marginal value is not constant (Corollary 
2). The scaling coefficient $\rho$ can be elicited by asking a midway level $y$ such that a change from the least preferred level of $0 \mathrm{~m}^{3}$ to level $y \mathrm{~m}^{3}$ is equally preferred to a change from the level $y \mathrm{~m}^{3}$ to the most preferred level of $50 \cdot 200 \mathrm{~m}^{3}=10000 \mathrm{~m}^{3}$. Specifying $y=700$ results to the exponential value functions $v_{2}$ and $V_{2}$.

The criterion specific portfolio value functions $V_{3}, V_{4}$ and $V_{5}$ can be elicited with techniques of Section 4.1. The DM uses the difference standard technique for criterion 'Natural water economy' and establishes that the successive changes between portfolios $x_{J 3}^{0},\langle 3$, exc. $\rangle,\langle 18$, exc. $\rangle$ and $\langle 45$, exc. $\rangle$ are equally preferred. Use of linear interpolation/extrapolation to obtain the weighting function values $w_{3}(k), k \in J \backslash\{3,18,45\}$, yields $V_{3}$ presented in Figure 5. For criterion 'Closest natural reserve' the DM states that adding a site to the portfolio that is right next to a natural reserve is equally preferred to adding two sites, both in a distance of $7.2 \mathrm{~km}$ from a natural reserve. The statement does not dependent on how close the other sites in the portfolio are to a natural reserve, wherefore it implies

$$
\langle k, 0 \mathrm{~km} ; 1,0 \mathrm{~km}\rangle \sim\langle k, 0 \mathrm{~km} ; 2,7.2 \mathrm{~km}\rangle \forall k \in\{0, \ldots ., m-2\} .
$$

Equation (9) implies $w_{5}(k+2)-w_{5}(k+1)=r_{5}\left[w_{5}(k+1)-w_{5}(k)\right]$ for all $k=0, \ldots, m-2$, where $r_{5}=\left(1 / v_{5}(7.2)-1\right)^{2}=1.1$, and hence $V_{5}$ is multiplicative (Corollary 1$)$.

The absolute values of $V_{1}, \ldots, V_{5}$ can be established with the methods of Section 4.2. For instance, the comparison of criteria 'Area' and 'Endangered species' can be operationalized by asking the DM to specify the number of species $y \in X_{4}$ such that

$$
\begin{aligned}
(\langle 10,5 \text { ha }\rangle,\langle 9,100 \text { species }\rangle) & \leftarrow(\langle 9,5 \text { ha }\rangle,\langle 9,100 \text { species }\rangle) \sim_{D} \\
(\langle 9,5 \text { ha }\rangle,\langle 9,100 \text { species } ; 1, y \text { species }\rangle) & \leftarrow(\langle 9,5 \text { ha }\rangle,\langle 9,100 \text { species }\rangle) .
\end{aligned}
$$

The answer $y=51$ results in the constraint $w_{1}(10)-w_{1}(9)=v_{4}(51)\left[w_{4}(10)-w_{4}(9)\right]$, where $v_{4}(51)=$ 0.53 (see Equation (11)). The elicited $w_{1}$ and $w_{4}$ in Figure 5 satisfy $w_{1}(10)-w_{1}(9)=w_{1}(50) / 50$ and $w_{4}(10)-w_{4}(9) \approx 0.0223 w_{4}(50)$, wherefore the constraint is roughly equivalent to $w_{1}(50) \approx$ $0.6 w_{4}(50)$ (cf. Figure 5 where $w_{1}(50)=0.15$ and $\left.w_{4}(50)=0.25\right)$. A similar comparison of criteria 

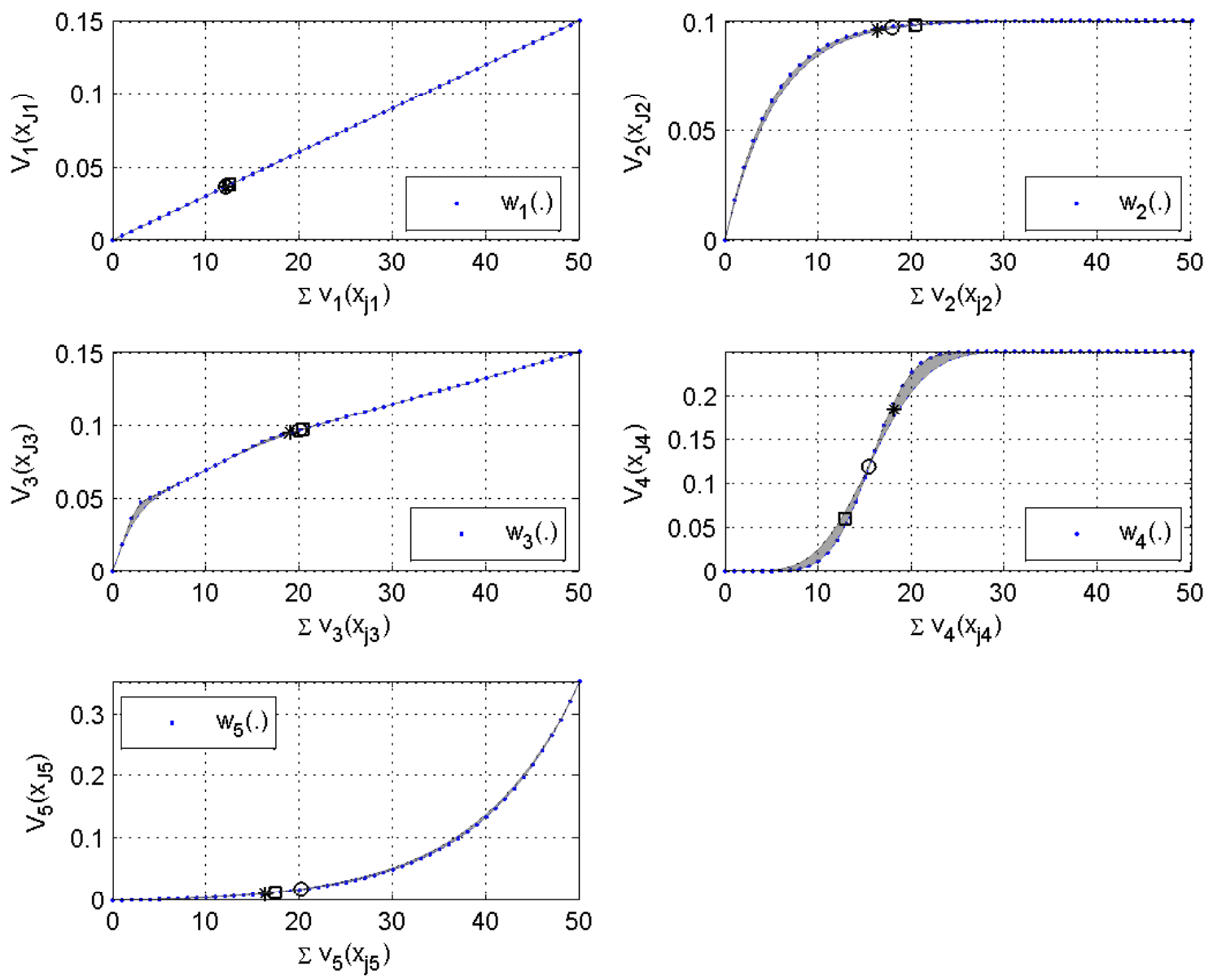

Figure 5 Criterion specific portfolio value functions $V_{1}, \ldots, V_{5}$. The grey area represents the set $\left\{\left(\sum_{j=1}^{m} v_{i}\left(x_{j i}\right), V_{i}\left(x_{J i}\right)\right) \mid v_{i}\left(x_{j i}\right) \in[0,1], j=1, \ldots, 50\right\}$ and the black points correspond to $\left(k, w_{i}(k)\right)$ for $k=0, \ldots, m$. The criterion specific portfolio values $V_{i}\left(\hat{x}\left(z^{*}\right)\right), V_{i}\left(\hat{x}\left(z^{a}\right)\right)$ and $V_{i}\left(\hat{x}\left(z^{b}\right)\right)$ are marked with asterisks, circles, and squares, respectively.

$i=2,3,5$ and criterion $i^{\prime}=1$ results in 3 additional constraints that together with the requirement $V\left(x^{*}\right)=\sum_{i=1}^{5} w_{i}(50)=1$ define the absolute values of $w_{i}$.

Table 2 contains a randomly generated data set of 50 sites to test the optimization models. The performance levels $x_{j i}^{g o}$ are uniformly distributed on each measurement scale and $x_{j i}^{n o}=x_{i}^{0}$ for all $j=1, . ., 50, i=1, . ., 5$. The cost $a_{j}$ of acquiring site $j$ for conservation is obtained by generating a random per hectare cost uniformly distributed between 80 and 120 euros for each site, and then 
multiplying it with the area of the site $x_{j 1}^{g o}$. The total budget is $b=7083$ euros, which corresponds to $50 \%$ of the sum of all sites' costs.

The optimal portfolio $z^{*}$ was solved with a Java implementation of the implicit enumeration algorithm (Section 5.1) in less than 2 seconds with a standard laptop (2.53 GHz, 4GB memory). The overall value of $z^{*}$ is $V\left(\hat{x}\left(z^{*}\right)\right)=0.422$, and the criterion specific portfolio values and the sums of the projects' scores are shown in Figure 5. The MILP model $(20)-(28)$ in which $V_{2}, \ldots, V_{5}$ were approximated by piecewise linear extensions of weighting functions (i.e., $\chi^{i}=(0,1, \ldots, m)^{T}$, $\left.\tilde{V}_{i}\left(\chi_{d}^{i}\right)=w_{i}\left(\chi_{d}^{i}\right), i=2, \ldots, 5, d=1, \ldots, m+1\right)$ produced the same optimal solution $z^{*}$ in less than a second.

For comparison, we also computed optimal solutions for the additive-linear model $\max _{z \in Z_{F}} \sum_{j=1}^{50} z_{j} \sum_{i=1}^{5} \tilde{w}_{i} v_{i}\left(x_{j i}^{g o}\right)$ with two sets of criterion weights $\tilde{w}$. First, $z^{a}$ denotes the optimal portfolio when the weights capture the entire 'portfolio value swings' $x_{J i}^{*} \leftarrow x_{J i}^{0}$, i.e, $\tilde{w}=\left(w_{1}(50), \ldots, w_{5}(50)\right)=(0.15,0.1,0.15,0.25,0.35)$. Second, $z^{b}$ denotes the optimal portfolio when the weights correspond to the 'project value swings' $\left(x_{1 i}^{*}, x_{2 i}^{0}, \ldots, x_{50 i}^{0}\right) \leftarrow x_{J i}^{0}$, i.e, $\tilde{w}=$ $\left(w_{1}(1), \ldots, w_{5}(1)\right) / \sum_{i} w_{i}(1) \approx(0.08,0.46,0.46,0.00,0.01)$. The additive-multilinear overall values were $V\left(\hat{x}\left(z^{a}\right)\right)=0.366$ and $V\left(\hat{x}\left(z^{b}\right)\right)=0.305$, which correspond to $83 \%$ and $72 \%$ of the optimal value of $V\left(\hat{x}\left(z^{*}\right)\right)=0.422$. The compositions of $z^{a}$ and $z^{b}$ are presented in Table 2 , and the criterion specific values $V_{i}\left(\hat{x}_{J i}\left(z^{a}\right)\right)$ and $V_{i}\left(\hat{x}_{J i}\left(z^{b}\right)\right)$ in Figure 5 . The number of different project specific decisions between $z^{*}$ and $z^{a}$ is 13 , and 18 between $z^{*}$ and $z^{b}$. This exemplifies that applying the additive-linear model in case where the assumption of constant marginal criterion specific portfolio value does not hold can lead to substantial value loss and erroneous project specific decisions.

\section{Discussion}

The developed portfolio value functions can be readily integrated with existing project portfolio selection models and processes based on the additive-linear portfolio value function. The phases of structuring objectives, developing criteria to measure the achievement of these objectives and eliciting criterion specific project value functions (i.e., project scoring) remain unchanged. For 
Table 2 Projects' performances and costs

\begin{tabular}{|c|c|c|c|c|c|c|c|c|c|}
\hline$j=1$ & $x_{j 1}^{g o}$ & $x_{j 2}^{g o}$ & $x_{j 3}^{g o}$ & $x_{j 4}^{g o}$ & $x_{j 5}^{g o}$ & $a_{j}$ & $z_{j}^{*}$ & $z_{j}^{a}$ & $z_{j}^{b}$ \\
\hline 1 & 2.3 & 173 & Poor & 18 & 0.7 & 213 & 0 & 1 & 1 \\
\hline 2 & 4.1 & 72 & Poor & 32 & 6.1 & 438 & 0 & 0 & 0 \\
\hline 3 & 1.7 & 156 & None & 9 & 7.5 & 139 & 0 & 1 & 1 \\
\hline 4 & 3.3 & 172 & Exc. & 46 & 2.8 & 329 & 1 & 1 & 1 \\
\hline 5 & 3.7 & 195 & None & 67 & 9.4 & 425 & 1 & 0 & 0 \\
\hline 6 & 0.8 & 114 & Exc. & 26 & 9.1 & 92 & 1 & 1 & 1 \\
\hline 7 & 0.9 & 72 & Good & 69 & 10 & 106 & 1 & 1 & 1 \\
\hline 8 & 1.6 & 127 & Exc. & 67 & 1.3 & 157 & 1 & 1 & 1 \\
\hline 9 & 2.1 & 155 & Good & 56 & 9.1 & 186 & 1 & 1 & 1 \\
\hline 10 & 4.2 & 180 & Poor & 69 & 7.8 & 440 & 1 & 0 & 1 \\
\hline 11 & 3.5 & 126 & Poor & 36 & 10 & 345 & 0 & 0 & 0 \\
\hline 12 & 0.6 & 110 & Poor & 53 & 3.2 & 68 & 1 & 1 & 1 \\
\hline 13 & 1.8 & 47 & Exc. & 23 & 8 & 147 & 1 & 1 & 1 \\
\hline 14 & 2.1 & 140 & Poor & 8 & 6.6 & 233 & 0 & 1 & 1 \\
\hline 15 & 1.5 & 34 & Good & 13 & 8.2 & 122 & 1 & 1 & 1 \\
\hline 16 & 3 & 180 & None & 42 & 7.9 & 289 & 1 & 0 & 1 \\
\hline 17 & 1.5 & 59 & Good & 99 & 6.3 & 125 & 1 & 1 & 1 \\
\hline 18 & 2.2 & 78 & Good & 47 & 0.1 & 187 & 1 & 1 & 1 \\
\hline 19 & 1.4 & 59 & None & 48 & 0.3 & 165 & 1 & 1 & 0 \\
\hline 20 & 4.2 & 108 & Good & 25 & 1.3 & 342 & 0 & 1 & 1 \\
\hline 21 & 3 & 148 & Poor & 76 & 9.2 & 325 & 1 & 1 & 1 \\
\hline 22 & 5 & 39 & Exc. & 82 & 5.5 & 549 & 1 & 0 & 0 \\
\hline 23 & 3.6 & 39 & Good & 96 & 6.2 & 383 & 1 & 1 & 0 \\
\hline 24 & 0.5 & 119 & Good & 77 & 4.9 & 52 & 1 & 1 & 1 \\
\hline 25 & 3 & 48 & None & 25 & 2.9 & 342 & 0 & 1 & 0 \\
\hline 26 & 2.5 & 49 & None & 53 & 3.2 & 279 & 1 & 1 & 0 \\
\hline 27 & 3.9 & 44 & Poor & 9 & 1.7 & 411 & 0 & 0 & 0 \\
\hline 28 & 2.9 & 165 & None & 80 & 7.6 & 343 & 1 & 1 & 0 \\
\hline 29 & 3 & 1 & Exc. & 92 & 9.3 & 292 & 1 & 1 & 1 \\
\hline 30 & 3 & 132 & Poor & 18 & 7.4 & 354 & 0 & 0 & 1 \\
\hline 31 & 4.2 & 73 & Poor & 47 & 8.6 & 490 & 0 & 0 & 0 \\
\hline 32 & 1.5 & 149 & Exc. & 62 & 7.7 & 138 & 1 & 1 & 1 \\
\hline 33 & 4.9 & 148 & Poor & 22 & 2.9 & 472 & 0 & 0 & 0 \\
\hline 34 & 4.1 & 192 & Exc. & 68 & 8.9 & 340 & 1 & 1 & 1 \\
\hline 35 & 4.7 & 184 & Poor & 10 & 5.9 & 442 & 0 & 0 & 1 \\
\hline 36 & 1.6 & 137 & Exc. & 51 & 3.4 & 163 & 1 & 1 & 1 \\
\hline 37 & 1.1 & 195 & Poor & 20 & 9.1 & 114 & 1 & 1 & 1 \\
\hline 38 & 2.9 & 4 & Good & 76 & 7.5 & 273 & 1 & 1 & 0 \\
\hline 39 & 0.6 & 91 & Exc. & 45 & 4.2 & 70 & 1 & 1 & 1 \\
\hline 40 & 4.2 & 21 & None & 36 & 1.4 & 452 & 0 & 0 & 0 \\
\hline 41 & 2.1 & 20 & Good & 31 & 4.4 & 249 & 0 & 1 & 0 \\
\hline 42 & 1.3 & 124 & None & 34 & 8.8 & 156 & 0 & 0 & 1 \\
\hline 43 & 2.1 & 28 & Exc. & 20 & 5.6 & 211 & 1 & 1 & 1 \\
\hline 44 & 3.5 & 197 & Poor & 29 & 2.3 & 409 & 0 & 1 & 1 \\
\hline 45 & 3 & 105 & Good & 36 & 5.4 & 318 & 0 & 1 & 1 \\
\hline 46 & 4.8 & 51 & Poor & 18 & 3.5 & 503 & 0 & 0 & 0 \\
\hline 47 & 4.6 & 124 & None & 25 & 8.5 & 473 & 0 & 0 & 0 \\
\hline 48 & 2 & 176 & Exc. & 34 & 4.8 & 170 & 1 & 1 & 1 \\
\hline 49 & 4.5 & 59 & Good & 59 & 2.4 & 516 & 1 & 0 & 0 \\
\hline 50 & 3.5 & 82 & Exc. & 30 & 8.6 & 332 & 0 & 0 & 1 \\
\hline
\end{tabular}

those criteria that have non-constant marginal value, a multilinear $V_{i}$ can be elicited with methods of Section 4.1. The choice among the additive, multiplicative or multilinear aggregation of the criterion specific portfolio values can be determined by examining which one of these forms best captures preferences over the performance levels of a single project $X_{1} \times \ldots \times X_{n}$. Hence, the additive-multilinear portfolio value function may well be adequate in most applications.

The elicitation burden can be reduced by eliciting only the weighting function values that are needed. For instance, if the value of not implementing a project is equal to $v_{i}\left(x_{i}^{0}\right)$ for all $i \in I$, then the weighting functions $w_{i}$ needs to be elicited only up to the point $m^{\prime}$ which is the maximum number of projects that can be included in any feasible portfolio, i.e., $m^{\prime}=\max \left\{\sum_{j=1}^{m} z_{j} \mid z \in Z_{F}\right\}$. This is because in Equation $(7)$ each $w_{i}(k)$ such that $k>m^{\prime}$ is always multiplied by $v_{i}\left(x_{i}^{0}\right)=0$. 
For instance, if there are $m=200$ project candidates, but at most 40 can be included in a feasible portfolio, then only the values $w_{i}(1), \ldots, w_{i}(40)$ need to be elicited. Furthermore, if $m^{\prime}$ is increased to $m^{\prime \prime}$ due to additional resources or new project candidates, it suffices to elicit the missing values $w_{i}\left(m^{\prime}+1\right), \ldots, w_{i}\left(m^{\prime \prime}\right)$.

The main results of this paper can be readily extended to decision making under uncertainty by applying the multiattribute utility theory (MAUT; Keeney and Raiffa 1976), which uses a single preference relation to compare probability distributions over multiattribute performance levels. In MAUT, a set of attributes is utility independent (UI) if preference order of probability distributions on the attribute set is not affected by the fixed level of other attributes. Utility independence implies the same restrictions on the utility function as weak difference independence does on the measurable value function (Dyer and Sarin 1979). Hence, Theorems 1-3 hold if the terms 'value function' and 'WDI' are replaced by 'utility function' and 'UI', respectively. This notwithstanding, elicitation questions that harness preferences between changes (e.g. standard difference technique) cannot be applied as such, but have to be replaced with comparisons of probability distributions. Furthermore, replacing the objective function in (18) with expected portfolio utility requires evaluating multidimensional integrals, unless the attribute specific performances are stochastically independent.

\section{Conclusions}

In this paper we have relaxed some common preference assumptions in multiobjective portfolio decision analysis. Specifically, if each criterion is weak difference independent, both as a project and portfolio performance measure, and preferences are independent of project indexing, then (i) the criterion specific portfolio values are captured by symmetric multilinear functions and (ii) these values can be aggregated with a (non-symmetric) multilinear function to obtain the overall portfolio value. Furthermore, if preferences over the performances of a single project are captured by an additive (a multiplicative) value function, then the overall portfolio value is an additive (a multiplicative) function of the symmetric multilinear criterion specific portfolio values. 
This paper suggests several avenues for future research. First, preference elicitation procedures need to be tested and further developed in real-life case-studies. Second, extending the value models to admit incomplete preference information by modeling sets of feasible weighting functions can be fruitful based on experiences from similar extensions to the additive-linear value model (Liesiö et al. 2007). Finally, it would be interesting to examine the implications of the relaxed preference assumptions to decision support models that prioritize projects based on their value-to-cost ratios.

\section{Appendix A: Weak Difference Independence}

The set of attributes $X_{K}, K \subseteq J \times I$, is WDI if

$$
\begin{aligned}
& \left(x_{K}^{a}, x_{\bar{K}}\right) \leftarrow\left(x_{K}^{b}, x_{\bar{K}}\right) \succeq_{D}\left(x_{K}^{c}, x_{\bar{K}}\right) \leftarrow\left(x_{K}^{d}, x_{\bar{K}}\right) \Rightarrow \\
& \left(x_{K}^{a}, x_{\bar{K}}^{\prime}\right) \leftarrow\left(x_{K}^{b}, x_{\bar{K}}^{\prime}\right) \succeq_{D}\left(x_{K}^{c}, x_{\bar{K}}^{\prime}\right) \leftarrow\left(x_{K}^{d}, x_{\bar{K}}^{\prime}\right) \forall x_{\bar{K}}^{\prime} \in X_{\bar{K}} .
\end{aligned}
$$

Evaluating $V$ over the set of attributes $X_{K}$ with other attributes $X_{\bar{K}}$ fixed at any levels $x_{\bar{K}}^{a}$ must imply exactly the same preferences $\succeq_{D}$ as evaluating $V$ with the other attributes at some other level $x_{\bar{K}}^{b} \in X_{\bar{K}}$ and hence

$$
V\left(x_{K}, x_{\bar{K}}^{a}\right)=\alpha\left(x_{\bar{K}}^{a}\right) V\left(x_{K}, x_{\bar{K}}^{b}\right)+\beta\left(x_{\bar{K}}^{a}\right) \forall x_{K} \in X_{K},
$$

where $\alpha, \beta$ depend only on attributes $X_{\bar{K}}$ and $\alpha>0$ (Dyer and Sarin 1979, Theorem 2). WDI extends naturally to situations where preferences over $X_{K}$ are independent of some attributes $X_{K^{\prime}}$, $K^{\prime} \subset \bar{K}$, but may depend on the other attributes $X_{K^{\prime \prime}}, K^{\prime \prime}=\bar{K} \backslash K^{\prime}$. More specifically, the set of attributes $X_{K}$ is conditionally WDI of attributes $X_{K^{\prime}}$, if for any fixed $x_{K^{\prime \prime}} \in X_{K^{\prime \prime}}$

$$
\begin{aligned}
& \left(x_{K}^{a}, x_{K^{\prime}}, x_{K^{\prime \prime}}\right) \leftarrow\left(x_{K}^{b}, x_{K^{\prime}}, x_{K^{\prime \prime}}\right) \succeq_{D}\left(x_{K}^{c}, x_{K^{\prime}}, x_{K^{\prime \prime}}\right) \leftarrow\left(x_{K}^{d}, x_{K^{\prime}}, x_{K^{\prime \prime}}\right) \Rightarrow \\
& \left(x_{K}^{a}, x_{K^{\prime}}^{\prime}, x_{K^{\prime \prime}}\right) \leftarrow\left(x_{K}^{b}, x_{K^{\prime}}^{\prime}, x_{K^{\prime \prime}}\right) \succeq_{D}\left(x_{K}^{c}, x_{K^{\prime}}^{\prime}, x_{K^{\prime \prime}}\right) \leftarrow\left(x_{K}^{d}, x_{K^{\prime}}^{\prime}, x_{K^{\prime \prime}}\right) \forall x_{K^{\prime}}^{\prime} \in X_{K^{\prime}} .
\end{aligned}
$$

If $X_{K}$ is conditionally WDI of $X_{K^{\prime}}$, then for any $x_{K^{\prime \prime}} \in X_{K^{\prime \prime}} V$ must satisfy

$$
V\left(x_{K}, x_{K^{\prime}}^{a}, x_{K^{\prime \prime}}\right)=\alpha\left(x_{K^{\prime}}^{a}\right) V\left(x_{K}, x_{K^{\prime}}^{b}, x_{K^{\prime \prime}}\right)+\beta\left(x_{K^{\prime}}^{a}\right) \forall x_{K} \in X_{K},
$$

where $\alpha, \beta$ depend only on attributes $X_{K^{\prime}}$ and $\alpha>0$ (cf. conditional utility independence; Keeney and Raiffa 1976). 


\section{Appendix B: Properties of the Symmetric Multilinear Criterion Specific Portfolio Value}

Among portfolios that have the sum of the projects' scores equal to $s$, the greatest and lowest $V_{i}$ are obtained by portfolios with the composition $\left\langle k_{1}, x_{i}^{*} ; k_{2}, y\right\rangle$ for some $k_{1}, k_{2} \geq 0$ and $y \in X_{i}$ such that $k_{1} v_{i}\left(x_{i}^{*}\right)+k_{2} v_{i}(y)=k_{1}+k_{2} v_{i}(y)=s$.

Theorem 5. Let $i \in I$ and $x_{J i} \in X_{J i}$. Then $V_{i}\left(x_{J i}\right) \in\left[\underline{V}_{i}\left(\sum_{j=1}^{m} v_{i}\left(x_{j i}\right)\right), \bar{V}_{i}\left(\sum_{j=1}^{m} v_{i}\left(x_{j i}\right)\right)\right]$, where

$$
\begin{aligned}
& \underline{V}_{i}(s):=\min _{k_{1}, k_{2} \in \mathbb{Z}}\left\{V_{i}\left(\left\langle k_{1}, x_{i}^{*} ; k_{2}, y\right\rangle\right) \mid k_{1} \in[0, s], k_{2} \in\left[s-k_{1}, m-k_{1}\right], v_{i}(y)=\left(s-k_{1}\right) / k_{2}\right\} \\
& \bar{V}_{i}(s):=\max _{k_{1}, k_{2} \in \mathbb{Z}}\left\{V_{i}\left(\left\langle k_{1}, x_{i}^{*} ; k_{2}, y\right\rangle\right) \mid k_{1} \in[0, s], k_{2} \in\left[s-k_{1}, m-k_{1}\right], v_{i}(y)=\left(s-k_{1}\right) / k_{2}\right\} .
\end{aligned}
$$

Using Equation (7) to evaluate the $V_{i}$ in these optimization problems requires the enumeration of all $2^{m}-1$ subsets $J^{\prime} \subseteq J$, which is not practical since often in applications the number of projects $m$ can be in the dozens. However, an alternative form for $(7)$ is $V_{i}\left(x_{J i}\right)=\sum_{k=0}^{m} w_{i}(k) F_{k}^{i}\left(x_{J i}\right)$, where the terms

$$
F_{k}^{i}\left(x_{J i}\right):=\sum_{\substack{J^{\prime} \subseteq J \\\left|J^{\prime}\right|=k}} \prod_{j \in J^{\prime}} v_{i}\left(x_{j i}\right) \prod_{j \notin J^{\prime}}\left(1-v_{i}\left(x_{j i}\right)\right)
$$

depend only on the project scores $v_{i}\left(x_{j i}\right)$ and can be evaluated with a computational effort proportional to $m^{2}$ (for details see, e.g., Chen and Liu (1997)).

Using this form in the additive-multilinear portfolio value (5) results in $V(x)=$ $\sum_{i=1}^{n} \sum_{k=0}^{m} w_{i}(k) F_{k}^{i}\left(x_{J i}\right)$, wherefore any statement that two changes are equally preferred corresponds to a linear constraint on the weighting function values $w_{i}(0), \ldots, w_{i}(m), i \in I$, i.e.,

$$
x^{a} \leftarrow x^{b} \sim_{D} x^{c} \leftarrow x^{d} \Leftrightarrow \sum_{i=1}^{n} \sum_{k=1}^{m} w_{i}(k)\left[F_{k}^{i}\left(x_{J i}^{a}\right)-F_{k}^{i}\left(x_{J i}^{b}\right)-F_{k}^{i}\left(x_{J i}^{c}\right)+F_{k}^{i}\left(x_{J i}^{d}\right)\right]=0
$$

for any $x^{a}, x^{b}, x^{c}, x^{d} \in X$.

\section{Appendix C: Proofs}

The proofs of Theorems 1-3 are listed in a reverse order since the proof of Theorem 1 builds on Theorem 2, and the proof of Theorem 2 buils on Theorem 3. The following lemma provides some general properties of multilinear mappings $M_{d}:[0,1]^{d} \rightarrow \mathbb{R}$ that are used throughout the proofs. 
Lemma 1. Let $d \in \mathbb{N}_{+}, S_{d}=\{1, \ldots, d\}, a \in[0,1]^{d}, b\left(S^{\prime}\right) \in \mathbb{R}_{+}$for all $S^{\prime} \subseteq S_{d}, c \in \mathbb{R}$ and

$$
M_{d}(a)=\sum_{S^{\prime} \subseteq S_{d}} b\left(S^{\prime}\right) \prod_{s \in S^{\prime}} a_{s} \prod_{s \in S_{d} \backslash S^{\prime}}\left(1-a_{s}\right) .
$$

(i) If $b\left(S^{\prime}\right)=1 \forall S^{\prime} \subseteq S$, then $M_{d}(a)=1$.

(ii) If $b\left(S^{\prime}\right)=\sum_{s \in S^{\prime}} b(\{s\}) \forall S^{\prime} \subseteq S_{d}, b(\emptyset)=0$, then $M_{d}(a)=\sum_{s \in S_{d}} a_{s} b(\{s\})$.

(iii) If $1+c b\left(S^{\prime}\right)=\prod_{s \in S^{\prime}}[1+c b(\{s\})] \forall S^{\prime} \subseteq S_{d}, b(\emptyset)=0$, then $1+c M_{d}(a)=\prod_{s \in S_{d}}\left[1+a_{s} c b(\{s\})\right]$.

(iv) If $b\left(S^{\prime}\right)>b\left(S^{\prime \prime}\right) \forall S^{\prime \prime} \subset S^{\prime} \subseteq S_{d}$, then $M_{d}$ is strictly increasing and linear in each $a_{s}$.

Proof. (i)-(iii) are proved by induction using the following result: Let $S^{\prime} \subseteq S_{d}$ and denote $A_{d}\left(S^{\prime}\right):=\prod_{s \in S^{\prime}} a_{s} \prod_{s \in S_{d} \backslash S^{\prime}}\left(1-a_{s}\right)$. Then $M_{d}(a)=\sum_{S^{\prime} \subseteq S_{d}} b\left(S^{\prime}\right) A_{d}\left(S^{\prime}\right)=\sum_{S^{\prime} \subseteq S_{d}, d \in S^{\prime}} b\left(S^{\prime}\right) A_{d}\left(S^{\prime}\right)+$ $\sum_{S^{\prime} \subseteq S_{d}, d \notin S^{\prime}} b\left(S^{\prime}\right) A_{d}\left(S^{\prime}\right)=\sum_{S^{\prime} \subseteq S_{d-1}} b\left(S^{\prime} \cup\{d\}\right) A_{d}\left(S^{\prime} \cup\{d\}\right)+\sum_{S^{\prime} \subseteq S_{d-1}} b\left(S^{\prime}\right) A_{d}\left(S^{\prime}\right)=$ $\sum_{S^{\prime} \subseteq S_{d-1}} b\left(S^{\prime} \cup\{d\}\right) a_{d} A_{d-1}\left(S^{\prime}\right)+\sum_{S^{\prime} \subseteq S_{d-1}} b\left(S^{\prime}\right)\left(1-a_{d}\right) A_{d-1}\left(S^{\prime}\right) \Leftrightarrow$

$$
M_{d}(a)=\sum_{S^{\prime} \subseteq S_{d-1}}\left[b\left(S^{\prime} \cup\{d\}\right) a_{d}+b\left(S^{\prime}\right)\left(1-a_{d}\right)\right] A_{d-1}\left(S^{\prime}\right) .
$$

(i) For $d=1, M_{1}(a)=\left(1-a_{1}\right)+a_{1}=1$. For $d>1$ (32) gives

$$
M_{d}(a)=\sum_{S^{\prime} \subseteq S_{d-1}}\left[a_{d}+\left(1-a_{d}\right)\right] A_{d-1}\left(S^{\prime}\right)=\sum_{S^{\prime} \subseteq S_{d-1}} A_{d-1}\left(S^{\prime}\right)=M_{d-1}(a) .
$$

(ii) For $d=1, M_{1}(a)=b(\{1\}) a_{1}+b(\emptyset)\left(1-a_{1}\right)=a_{1} b(\{1\})$. For $d>1$ (32) gives

$$
\begin{aligned}
M_{d}(a) & =\sum_{S^{\prime} \subseteq S_{d-1}}\left[\left(b\left(S^{\prime}\right)+b(\{d\})\right) a_{d}+b\left(S^{\prime}\right)\left(1-a_{d}\right)\right] A_{d-1}\left(S^{\prime}\right) \\
& =b(\{d\})) a_{d} \underbrace{\sum_{S^{\prime} \subseteq S_{d-1}} A_{d-1}\left(S^{\prime}\right)}_{=1}+\underbrace{\sum_{S^{\prime} \subseteq S_{d-1}} b\left(S^{\prime}\right) A_{d-1}\left(S^{\prime}\right)}_{=M_{d-1}(a)}=b(\{d\})) a_{d}+M_{d-1}(a) .
\end{aligned}
$$

(iii) For $d=1,1+c M_{1}(a)=1+c\left[b(\{1\}) a_{1}+b(\emptyset)\left(1-a_{1}\right)\right]=1+c b(\{1\}) a_{1}$. For $d>1$ (32) gives

$$
\begin{aligned}
1+c M_{d}(a) & =\overbrace{\sum_{S^{\prime} \subseteq S_{d-1}} A_{d-1}\left(S^{\prime}\right)}^{=1}+\sum_{\substack{S^{\prime} \subseteq S_{d-1} \\
=c b\left(S^{\prime} \cup\{d\}\right) a_{d}}}\left[c b\left(S^{\prime} \cup\{d\}\right) a_{d}+c b\left(S^{\prime}\right)\left(1-a_{d}\right)\right] A_{d-1}\left(S^{\prime}\right) \\
& =\sum_{S^{\prime} \subseteq S_{d-1}}[\overbrace{(1+c b(\{d\}))\left(1+c b\left(S^{\prime}\right)\right) a_{d}-a_{d}}+1+\left(1-a_{d}\right) c b\left(S^{\prime}\right)] A_{d-1}\left(S^{\prime}\right) \\
& =\sum_{S^{\prime} \subseteq S_{d-1}}\left[(1+c b(\{d\})) a_{d}+1-a_{d}\right]\left(1+c b\left(S^{\prime}\right)\right) A_{d-1}\left(S^{\prime}\right)
\end{aligned}
$$




$$
=\left[1+c b(\{d\}) a_{d}\right][\underbrace{\sum_{S^{\prime} \subseteq S_{d-1}} A_{d-1}\left(S^{\prime}\right)}_{=1}+c \underbrace{\sum_{S^{\prime} \subseteq S_{d-1}} b\left(S^{\prime}\right) A_{d-1}\left(S^{\prime}\right)}_{=M_{d-1}(a)}] .
$$

(iv) From (32) it is easy to see that for any $t \in S_{d}$

$$
\begin{aligned}
& M_{d}(a)=\sum_{S^{\prime} \subseteq\left(S_{d} \backslash\{t\}\right)}\left[b\left(S^{\prime} \cup\{t\}\right) a_{t}+b\left(S^{\prime}\right)\left(1-a_{t}\right)\right] \prod_{s \in S^{\prime}} a_{s} \prod_{s \in S_{d} \backslash\{t\} \backslash S^{\prime}}\left(1-a_{s}\right) \\
& =a_{t} \underbrace{\sum_{S^{\prime} \subseteq\left(S_{d} \backslash\{t\}\right)}\left[b\left(S^{\prime} \cup\{t\}\right)-b\left(S^{\prime}\right)\right] \prod_{s \in S^{\prime}} a_{s} \prod_{s \in S_{d} \backslash\{t\} \backslash S^{\prime}}\left(1-a_{s}\right)}_{=\alpha\left(a_{1}, \ldots, a_{t-1}, a_{t+1}, \ldots, a_{d}\right)}+\underbrace{\sum_{S^{\prime} \subseteq(S \backslash\{t\})} b\left(S^{\prime}\right) \prod_{s \in S^{\prime}} a_{s} \prod_{s \in S_{d} \backslash\{t\} \backslash S^{\prime}}\left(1-a_{s}\right)}_{=\beta\left(a_{1}, \ldots, a_{t-1}, a_{t+1}, \ldots, a_{d}\right)},
\end{aligned}
$$

where $\alpha>0$, since $\left[b\left(S^{\prime} \cup\{t\}\right)-b\left(S^{\prime}\right)\right]>0$.

\section{C.1. Proof of Theorem 3}

' $\Rightarrow$ ': Let $V\left(x^{0}\right)=0$ and $V\left(x^{*}\right)=1$. Since each $X_{J i}$ is WDI (Assumption 3), (29) gives

$$
V\left(x_{J i}, x_{J \bar{i}}\right)=\alpha\left(x_{J \bar{i}}\right) V\left(x_{J i}, x_{J \bar{i}}^{0}\right)+\beta\left(x_{J \bar{i}}\right), \forall\left(x_{J i}, x_{J \bar{i}}\right) \in X .
$$

Evaluating (33) at $x_{J i}=x_{J i}^{0}$ gives $V\left(x_{J i}^{0}, x_{J \bar{i}}\right)=\alpha\left(x_{J \bar{i}}\right) V\left(x^{0}\right)+\beta\left(x_{J \bar{i}}\right)=\beta\left(x_{J \bar{i}}\right)$. Evaluating (33) at $x_{J i}=x_{J i}^{*}$ gives $V\left(x_{J i}^{*}, x_{J \bar{i}}\right)=\alpha\left(x_{J \bar{i}}\right) V\left(x_{J i}^{*}, x_{J \bar{i}}^{0}\right)+\beta\left(x_{J \bar{i}}\right)$, i.e, $\alpha\left(x_{J \bar{i}}\right)=\left[V\left(x_{J i}^{*}, x_{J \bar{i}}\right)-\right.$ $\left.\beta\left(x_{J \bar{i}}\right)\right] / V\left(x_{J i}^{*}, x_{J \bar{l}}^{0}\right)$. Substituting $\alpha$ and $\beta$ into (33) and denoting $V_{i}\left(x_{J i}\right):=V\left(x_{J i}, x_{J \bar{i}}^{0}\right)$ results in

$$
\begin{aligned}
V\left(x_{J i}, x_{J \bar{i}}\right) & =\frac{V\left(x_{J i}^{*}, x_{J \bar{i}}\right)-V\left(x_{J i}^{0}, x_{J \bar{l}}\right)}{V_{i}\left(x_{J i}^{*}\right)} V_{i}\left(x_{J i}\right)+V\left(x_{J i}^{0}, x_{J \bar{i}}\right) \\
& =\frac{V_{i}\left(x_{J i}\right)}{V_{i}\left(x_{J i}^{*}\right)} V\left(x_{J i}^{*}, x_{J \bar{i}}\right)+\left(1-\frac{V_{i}\left(x_{J i}\right)}{V_{i}\left(x_{J i}^{*}\right)}\right) V\left(x_{J i}^{0}, x_{J \bar{i}}\right) .
\end{aligned}
$$

Applying (34) repeatedly for each $i=1, \ldots, n$ to decompose $V(x)$ gives

$$
\begin{aligned}
V(x)= & \frac{V_{1}\left(x_{J 1}\right)}{V_{1}\left(x_{J 1}^{*}\right)} V\left(x_{J 1}^{*}, x_{J \overline{1}}\right)+\left(1-\frac{V_{1}\left(x_{J 1}\right)}{V_{1}\left(x_{J 1}^{*}\right)}\right) V\left(x_{J 1}^{0}, x_{J \overline{1}}\right) \\
= & \frac{V_{1}\left(x_{J 1}\right)}{V_{1}\left(x_{J 1}^{*}\right)} \frac{V_{1}\left(x_{J 2}\right)}{V_{1}\left(x_{J 2}^{*}\right)} V\left(x_{J 1}^{*}, x_{J 2}^{*}, x_{J \overline{12}}\right)+\frac{V_{1}\left(x_{J 1}\right)}{V_{1}\left(x_{J 1}^{*}\right)}\left(1-\frac{V_{2}\left(x_{J 2}\right)}{V_{2}\left(x_{J 2}^{*}\right)}\right) V\left(x_{J 1}^{*}, x_{J 2}^{0}, x_{J \overline{12}}\right) \\
& +\left(1-\frac{V_{1}\left(x_{J 1}\right)}{V_{1}\left(x_{J 1}^{*}\right)}\right) \frac{V_{1}\left(x_{J 2}\right)}{V_{1}\left(x_{J 2}^{*}\right)} V\left(x_{J 1}^{0}, x_{J 2}^{*}, x_{J \overline{1}}\right)+\left(1-\frac{V_{1}\left(x_{J 1}\right)}{V_{1}\left(x_{J 1}^{*}\right)}\right)\left(1-\frac{V_{2}\left(x_{J 2}\right)}{V_{2}\left(x_{J 2}^{*}\right)}\right) V\left(x_{J 1}^{0}, x_{J 2}^{0}, x_{J \overline{12}}\right) \\
& \ldots \\
= & \sum_{I^{\prime} \subseteq I}\left[\prod_{i \in I^{\prime}} \frac{V_{i}\left(x_{J i}\right)}{V_{i}\left(x_{J i}^{*}\right)} \prod_{i \notin I^{\prime}}\left(1-\frac{V_{i}\left(x_{J i}\right)}{V_{i}\left(x_{J i}^{*}\right)}\right) V\left(x_{J I^{\prime}}^{*}, x_{J \overline{I^{\prime}}}^{0}\right)\right] .
\end{aligned}
$$


Denoting $\lambda\left(I^{\prime}\right)=V\left(x_{J I^{\prime}}^{*}, x_{\overline{J I^{\prime}}}^{0}\right)$ implies $I^{\prime \prime} \subset I^{\prime} \Rightarrow \lambda\left(I^{\prime \prime}\right)<\lambda\left(I^{\prime}\right), V\left(x^{0}\right)=\lambda(\emptyset)=0, V\left(x^{*}\right)=\lambda(I)=1$.

Since each $X_{j i}$ is WDI (Assumption 2), applying (29) to $V_{i}\left(x_{J i}\right)=V_{i}\left(x_{j i}, x_{\bar{j} i}\right)=V\left(x_{j i}, x_{\bar{j} i}, x_{J \bar{i}}^{0}\right)$ gives $V\left(x_{j i}, x_{\bar{j} i}, x_{J \bar{i}}^{0}\right)=\alpha\left(x_{\bar{j} i}, x_{J \bar{i}}^{0}\right) V\left(x_{j i}, x_{\bar{j} i}^{0}, x_{J \bar{i}}^{0}\right)+\beta\left(x_{\bar{j} i}, x_{J \bar{l}}^{0}\right), \forall\left(x_{j i}, x_{\bar{j} i}\right) \in X_{J i} \Leftrightarrow$

$$
V_{i}\left(x_{j i}, x_{\bar{j} i}\right)=\alpha\left(x_{\bar{j} i}, x_{J \bar{i}}^{0}\right) V_{i}\left(x_{j i}, x_{\bar{j} i}^{0}\right)+\beta\left(x_{\bar{j} i}, x_{J \bar{i}}^{0}\right), \forall\left(x_{j i}, x_{\bar{j} i}\right) \in X_{J i} .
$$

Evaluating (35) at $x_{j i}=x_{i}^{0}$ gives $V_{i}\left(x_{j i}^{0}, x_{\bar{j} i}\right)=\alpha\left(x_{\bar{j} i}, x_{J \bar{i}}^{0}\right) V_{i}\left(x_{j i}^{0}, x_{\bar{j} i}^{0}\right)+\beta\left(x_{\bar{j} i}, x_{J \bar{i}}^{0}\right)=\beta\left(x_{\bar{j} i}, x_{J \bar{i}}^{0}\right)$. Evaluating (35) at $x_{j i}=x_{i}^{*}$ gives $V_{i}\left(x_{j i}^{*}, x_{\bar{j} i}\right)=\alpha\left(x_{\bar{j} i}, x_{J_{\bar{i}}}^{0}\right) V_{i}\left(x_{j i}^{*}, x_{\bar{j} i}^{0}\right)+\beta\left(x_{\bar{j} i}, x_{J \bar{i}}^{0}\right)$ i.e., $\alpha\left(x_{\bar{j} i}, x_{J \bar{i}}^{0}\right)=$ $\left[V_{i}\left(x_{j i}^{*}, x_{\overline{j i}}\right)-\beta\left(x_{\bar{j} i}, x_{J \bar{i}}^{0}\right)\right] / V_{i}\left(x_{j i}^{*}, x_{\bar{j} i}^{0}\right)$. Substituting $\alpha$ and $\beta$ into (35) gives

$$
V_{i}\left(x_{J i}\right)=\frac{V_{i}\left(x_{j i}, x_{\bar{j} i}^{0}\right)}{V_{i}\left(x_{j i}^{*}, x_{\bar{j} i}^{0}\right)} V_{i}\left(x_{j i}^{*}, x_{\bar{j} i}\right)+\left(1-\frac{V_{i}\left(x_{j i}, x_{\bar{j} i}^{0}\right)}{V_{i}\left(x_{j i}^{*}, x_{\bar{j} i}^{0}\right)}\right) V_{i}\left(x_{j i}^{0}, x_{\bar{j} i}\right),
$$

where $V_{i}\left(x_{j i}, x_{\bar{j} i}^{0}\right)=V_{i}\left(x_{j^{\prime} i}, x_{\bar{j}^{\prime} i}^{0}\right)$ for any $x_{j i}=x_{j^{\prime} i}, j, j^{\prime} \in J$ since the portfolios $\left(x_{j^{\prime} i}, x_{\bar{j}^{\prime} i}^{0}, x_{J \bar{i}}^{0}\right)$ and $\left(x_{j i}, x_{\bar{j} i}^{0}, x_{J \bar{i}}^{0}\right)$ are permutations of each other and thus project-symmetric preferences (Assumption 1) implies that they are equally preferred. Hence, we can define $v_{i}: X_{i} \rightarrow[0,1]$ as the function that satisfies $v_{i}\left(x_{j i}\right)=V_{i}\left(x_{j i}, x_{\bar{j} i}^{0}\right) / V_{i}\left(x_{j i}^{*}, x_{\bar{j} i}^{0}\right)$ for all $j \in J, x_{j i} \in X_{i}$. With this notation (36) becomes $V_{i}\left(x_{J i}\right)=v_{i}\left(x_{j i}\right) V_{i}\left(x_{j i}^{*}, x_{\bar{j} i}\right)+\left(1-v_{i}\left(x_{j i}\right)\right) V_{i}\left(x_{j i}^{0}, x_{\bar{j} i}\right)$, which can be used repeatedly for $j=1, \ldots, m$ to decompose $V_{i}\left(x_{J i}\right)$ into $V_{i}\left(x_{J i}\right)=\sum_{J^{\prime} \subseteq J} V_{i}\left(x_{J^{\prime} i}^{*}, x_{J^{\prime} i}^{0}\right)\left[\prod_{j \in J^{\prime}} v_{i}\left(x_{j i}\right) \prod_{j \notin J^{\prime}}\left(1-v_{i}\left(x_{j i}\right)\right)\right]$. Clearly, $\left(x_{J^{\prime} i}^{*}, x_{\overline{J^{\prime}} i}^{0}, x_{J^{\bar{i}}}^{0}\right)$ can be permutated to obtain any $\left(x_{J^{\prime \prime} i}^{*}, x_{\overline{J^{\prime \prime} i}}^{0}, x_{J_{\bar{i}}}^{0}\right)$ as long as $\left|J^{\prime \prime}\right|=\left|J^{\prime}\right|$. By Assumption 1 we may thus denote $w_{i}(k)=V_{i}\left(x_{J^{\prime} i}^{*}, x_{\overline{J^{\prime}} i}^{0}\right)$ for all $\left|J^{\prime}\right|=k, k \in\{0, \ldots, m\}$, and notice that $w_{i}(0)=V_{i}\left(x_{J i}^{0}\right)=V\left(x_{J i}^{0}, x_{J \bar{i}}^{0}\right)=V\left(x^{0}\right)=0, w_{i}(m)=V_{i}\left(x_{J i}^{*}\right)=V\left(x_{J i}^{*}, x_{J \bar{i}}^{0}\right)=\lambda(\{i\})$.

' $\Leftarrow$ ': Permutating rows of $x$ will not change the criterion specific values $V_{i}\left(x_{J i}\right)$, wherefore $V(x)$ remains constant and thus Assumption 1 holds. $V$ is linear in each $V_{i}$ and $V_{i}$ is linear in each $v_{i}$ (see property (iv) of Lemma 1), which implies that each $X_{J i}$ and $X_{j i}$ is WDI (Assumptions 2 and $3)$.

\section{C.2. Proof of Theorem 2}

' $\Rightarrow$ : Since Assumptions 1-3 hold, Theorem 3 implies that $V$ is multilinear (17). Choose arbitrary $j \in J, i^{\prime} \in I, I^{\prime} \subset I$ such that $i^{\prime} \notin I^{\prime}$ and denote $I^{\prime \prime}=I \backslash I^{\prime} \backslash\left\{i^{\prime}\right\}$. Since $X_{j \overline{i^{\prime}}}$ is conditionally WDI of $X_{j i^{\prime}}$ (Assumption 5), (30) implies

$$
V\left(x_{j I^{\prime}}^{*}, x_{j I^{\prime \prime}}^{0}, x_{j i^{\prime}}^{*}, x_{j I}^{0}\right)=\alpha\left(x_{j i^{\prime}}^{*}\right) V\left(x_{j I^{\prime}}^{*}, x_{j I^{\prime \prime}}^{0}, x_{j i^{\prime}}^{0}, x_{j I}^{0}\right)+\beta\left(x_{j i^{\prime}}^{*}\right) .
$$


If $I^{\prime}=\emptyset \quad(37)$ implies $\beta\left(x_{j i^{\prime}}^{*}\right)=V\left(x_{j i^{\prime}}^{*}, x_{j i^{\prime}}^{0}\right)=w_{i^{\prime}}(1)$. Evaluating (37) with $i^{\prime}=i_{2}, I^{\prime}=$ $\left\{i_{1}\right\}$ gives $V\left(x_{j i_{2}}^{*}, x_{j i_{1}}^{*}, x_{j I^{\prime \prime}}^{0}, x_{j I}^{0}\right)=\alpha\left(x_{j i_{2}}^{*}\right) w_{i_{1}}(1)+w_{i_{2}}(1)$ and with $i^{\prime}=i_{1}, I^{\prime}=\left\{i_{2}\right\}$ gives $V\left(x_{j i_{1}}^{*}, x_{j i_{2}}^{*}, x_{j I^{\prime \prime}}^{0}, x_{\bar{j} I}^{0}\right)=\alpha\left(x_{j i_{1}}^{*}\right) w_{i_{2}}(1)+w_{i_{1}}(1)$ (since $\left.V\left(x_{j i}^{*}, x_{\overline{j i}}^{0}\right)=w_{i}(1)\right)$. These together imply

$$
\frac{\alpha\left(x_{j i_{1}}^{*}\right)-1}{w_{i_{1}}(1)}=\frac{\alpha\left(x_{j i_{2}}^{*}\right)-1}{w_{i_{2}}(1)}=\kappa,
$$

for some $\kappa$. Since the choice of $i_{1}, i_{2}$ was arbitrary, this implies that for all $i^{\prime} \in I, \alpha\left(x_{j i}^{*}\right)=\kappa w_{i^{\prime}}(1)+1$.

Substituting $\alpha$ and $\beta$ into (37) gives

$$
V\left(x_{j I^{\prime}}^{*}, x_{j I^{\prime \prime}}^{0}, x_{j i^{\prime}}^{*}, x_{\bar{j} I}^{0}\right)=\left[\kappa w_{i^{\prime}}(1)+1\right] V\left(x_{j I^{\prime}}^{*}, x_{j I^{\prime \prime}}^{0}, x_{j i^{\prime}}^{0}, x_{\bar{j} I}^{0}\right)+w_{i^{\prime}}(1) .
$$

The left-hand side of (38) is equal to

$$
\begin{aligned}
& \sum_{\tilde{I} \subseteq I^{\prime} \cup\left\{i^{\prime}\right\}} \lambda(\tilde{I}) \prod_{i \in \tilde{I}} \frac{w_{i}(1)}{\lambda(\{i\})} \prod_{i \in I^{\prime} \cup\left\{i^{\prime}\right\}, i \notin \tilde{I}}\left(1-\frac{w_{i}(1)}{\lambda(\{i\})}\right) \\
= & \frac{w_{i^{\prime}}(1)}{\lambda\left(\left\{i^{\prime}\right\}\right)} \sum_{\tilde{I} \subseteq I^{\prime}} \lambda\left(\tilde{I} \cup\left\{i^{\prime}\right\}\right) \prod_{i \in \tilde{I}} \frac{w_{i}(1)}{\lambda(\{i\})} \prod_{i \in I^{\prime}, i \notin \tilde{I}}\left(1-\frac{w_{i}(1)}{\lambda(\{i\})}\right) \\
+ & \left(1-\frac{w_{i^{\prime}}(1)}{\lambda\left(\left\{i^{\prime}\right\}\right)}\right) \sum_{\tilde{I} \subseteq I^{\prime}} \lambda(\tilde{I}) \prod_{i \in \tilde{I}} \frac{w_{i}(1)}{\lambda(\{i\})} \prod_{i \in I^{\prime} i \notin \tilde{I}}\left(1-\frac{w_{i}(1)}{\lambda(\{i\})}\right)
\end{aligned}
$$

and the right-hand side is equal to

$$
\left[\kappa w_{i^{\prime}}(1)+1\right] \sum_{\tilde{I} \subseteq I^{\prime}} \lambda(\tilde{I}) \prod_{i \in \tilde{I}} \frac{w_{i}(1)}{\lambda(\{i\})} \prod_{i \in I^{\prime}, i \notin \tilde{I}}\left(1-\frac{w_{i}(1)}{\lambda(\{i\})}\right)+w_{i^{\prime}}(1) .
$$

Thus (38) is equivalent to

$$
\begin{aligned}
& \sum_{\tilde{I} \subseteq I^{\prime}}\left[\frac{w_{i^{\prime}}(1)}{\lambda\left(\left\{i^{\prime}\right\}\right)} \lambda\left(\tilde{I} \cup\left\{i^{\prime}\right\}\right)-\left(\frac{w_{i^{\prime}}(1)}{\lambda\left(\left\{i^{\prime}\right\}\right)}+\kappa w_{i^{\prime}}(1)\right) \lambda(\tilde{I})\right] \prod_{i \in \tilde{I}} \frac{w_{i}(1)}{\lambda(\{i\})} \prod_{i \in I, i \notin \tilde{I}}\left(1-\frac{w_{i}(1)}{\lambda(\{i\})}\right)=w_{i^{\prime}}(1) \\
& \sum_{\tilde{I} \subseteq I^{\prime}}\left[\lambda\left(\tilde{I} \cup\left\{i^{\prime}\right\}\right)-\left(1+\kappa \lambda\left(\left\{i^{\prime}\right\}\right)\right) \lambda(\tilde{I})\right] \prod_{i \in \tilde{I}} \frac{w_{i}(1)}{\lambda(\{i\})} \prod_{i \in I, i \notin \tilde{I}}\left(1-\frac{w_{i}(1)}{\lambda(\{i\})}\right)=\lambda\left(\left\{i^{\prime}\right\}\right)
\end{aligned}
$$

For $I^{\prime}=\left\{i^{\prime \prime}\right\}(39)$ gives

$$
\begin{aligned}
& {\left[\lambda\left(\left\{i^{\prime \prime}, i^{\prime}\right\}\right)-\left(1+\kappa \lambda\left(\left\{i^{\prime}\right\}\right)\right) \lambda\left(\left\{i^{\prime \prime}\right\}\right)\right] \frac{w_{i^{\prime \prime}}(1)}{\lambda\left(\left\{i^{\prime \prime}\right\}\right)}} \\
& +\left[\lambda\left(\left\{i^{\prime}\right\}\right)-\left(1+\kappa \lambda\left(\left\{i^{\prime}\right\}\right)\right) \lambda(\emptyset)\right]\left(1-\frac{w_{i^{\prime \prime}}(1)}{\lambda\left(\left\{i^{\prime \prime}\right\}\right)}\right)=\lambda\left(\left\{i^{\prime}\right\}\right) \\
& \Rightarrow \lambda\left(I^{\prime} \cup\left\{i^{\prime}\right\}\right)-\left(1+\kappa \lambda\left(\left\{i^{\prime}\right\}\right)\right) \lambda\left(I^{\prime}\right)=\lambda\left(\left\{i^{\prime}\right\}\right) .
\end{aligned}
$$


Thus, (40) holds for any $i^{\prime} \in I$ and $I^{\prime} \subset I$ such that $\left|I^{\prime}\right|=1$. Assuming that (40) holds for all $\left|I^{\prime}\right|<k<n$ and evaluating (39) for $\left|I^{\prime}\right|=k$ yields

$$
\begin{aligned}
& {\left[\lambda\left(I^{\prime} \cup\left\{i^{\prime}\right\}\right)-\left(1+\kappa \lambda\left(\left\{i^{\prime}\right\}\right)\right) \lambda\left(I^{\prime}\right)\right] \prod_{i \in I^{\prime}} \frac{w_{i}(1)}{\lambda(\{i\})}} \\
& +\sum_{\tilde{I} \subset I^{\prime}}\left[\lambda\left(\tilde{I} \cup\left\{i^{\prime}\right\}\right)-\left(1+\kappa \lambda\left(\left\{i^{\prime}\right\}\right)\right) \lambda(\tilde{I})\right] \prod_{i \in \tilde{I}} \frac{w_{i}(1)}{\lambda(\{i\})} \prod_{i \in I^{\prime}, i \notin \tilde{I}}\left(1-\frac{w_{i}(1)}{\lambda(\{i\})}\right) \\
& =\left[\lambda\left(I^{\prime} \cup\left\{i^{\prime}\right\}\right)-\left(1+\kappa \lambda\left(\left\{i^{\prime}\right\}\right)\right) \lambda\left(I^{\prime}\right)\right] \prod_{i \in I^{\prime}} \frac{w_{i}(1)}{\lambda(\{i\})}+\lambda\left(\left\{i^{\prime}\right\}\right) \sum_{\tilde{I} \subset I^{\prime}} \prod_{i \in \tilde{I}} \frac{w_{i}(1)}{\lambda(\{i\})} \prod_{i \in I^{\prime}, i \notin \tilde{I}}\left(1-\frac{w_{i}(1)}{\lambda(\{i\})}\right) \\
& =\left[\lambda\left(I^{\prime} \cup\left\{i^{\prime}\right\}\right)-\left(1+\kappa \lambda\left(\left\{i^{\prime}\right\}\right)\right) \lambda\left(I^{\prime}\right)\right] \prod_{i \in I^{\prime}} \frac{w_{i}(1)}{\lambda(\{i\})}+\lambda\left(\left\{i^{\prime}\right\}\right)\left(1-\prod_{i \in I^{\prime}} \frac{w_{i}(1)}{\lambda(\{i\})}\right)=\lambda\left(\left\{i^{\prime}\right\}\right),
\end{aligned}
$$

where $\sum_{\tilde{I} \subset I^{\prime}} \prod_{i \in \tilde{I}} \frac{w_{i}(1)}{\lambda(\{i\})} \prod_{i \in I^{\prime}, i \notin \tilde{I}}\left(1-\frac{w_{i}(1)}{\lambda(\{i\})}\right)=1-\prod_{i \in I^{\prime}} \frac{w_{i}\left(x_{J i}\right)}{\lambda(\{i\})}$ by property (i) of Lemma 1 . Thus by induction (40) holds for all $I^{\prime} \subset I$. If $\kappa=0$, (40) equals $\lambda\left(I^{\prime} \cup\left\{i^{\prime}\right\}\right)=\lambda\left(I^{\prime}\right)+\lambda\left(\left\{i^{\prime}\right\}\right)$ which can be used repeatedly to obtain $\lambda(\tilde{I})=\sum_{i \in \tilde{I}} \lambda(\{i\})$ for any $\tilde{I} \subseteq I$. Substituting this into the multilinear $V$ gives (property (ii), Lemma 1) $V(x)=\sum_{i=1}^{n} V_{i}(x)$, which is the additive-multilinear $V$. If $\kappa \neq 0$, (40) equals $1+\kappa \lambda\left(I^{\prime} \cup\left\{i^{\prime}\right\}\right)=\kappa(1+\kappa \lambda(\{i\})) \lambda\left(I^{\prime}\right)+1+\kappa \lambda\left(\left\{i^{\prime}\right\}\right)$ or equivalently $1+\kappa \lambda\left(I^{\prime} \cup\left\{i^{\prime}\right\}\right)=$ $(1+\kappa \lambda(\{i\}))\left(1+\kappa \lambda\left(I^{\prime}\right)\right)$, which can be used repeatedly obtain $1+\kappa \lambda(\tilde{I})=\prod_{i \in \tilde{I}}(\kappa \lambda(\{i\})+1)$ for all $\tilde{I} \subseteq I$. Substituting this into the multilinear portfolio value function results in (property (iii), Lemma 1 ) $1+\kappa V(x)=\prod_{i=1}^{n}\left[1+\kappa V_{i}(x)\right]$, which is the multiplicative-multilinear $V$.

' $\Leftarrow$ : Since the additive- and multiplicative-multilinear $V$ are special cases of multilinear $V$, Theorem 3 implies that Assumptions 1-3 hold. Also it is well known that additive and multiplicative value functions imply that each subset of the attributes is WDI (Dyer and Sarin 1979) and hence each $X_{J I^{\prime}}, I^{\prime} \subset I$ is WDI. This implies that each $X_{j I^{\prime}}$ is conditionally WDI of $X_{j \overline{I^{\prime}}}$ given $X_{\bar{j} I}$ (Assumption 5).

\section{C.3. Proof of Theorem 1}

We apply the following result in the proof: If the sets of attributes $Y^{1}, Y^{2} \subset X$ are both WDI and overlapping (i.e, $Y^{1} \cap Y^{2} \neq \emptyset$ and neither is a subset of the other), then the (set-theoretic) difference $Y^{1} \backslash Y^{2}$ is also WDI. This result was established in the context of multiattribute utility functions (Keeney and Raiffa 1976, Theorem 6.7) and later shown to apply for measurable value functions as well (Dyer and Sarin 1979, Corollary 2). 
$\Rightarrow$ : Let $I^{\prime} \subset I$ and denote $\{i(1), \ldots, i(k)\}=\overline{I^{\prime}}$. Then $X_{j I^{\prime}}$ can be constructed by a series of differences, where each set is WDI and overlapping, i.e, $X_{j I^{\prime}}=X_{j I} \backslash X_{J i(1)} \backslash X_{J i(2)} \backslash \cdots \backslash X_{J i(k)}$. The above result states that $X_{j I^{\prime}}$ is WDI, which implies that $X_{j I^{\prime}}$ is also conditionally WDI of $X_{j \overline{I^{\prime}}}$ given $X_{\bar{j} I}$ (Assumption 5). Thus, by Theorem $2 \mathrm{~V}$ is either additive- (5) or multiplicative-multilinear (6). Take arbitrary $i_{1}, i_{2} \in I$ and $j \in J$. Let $x_{j i_{1}}$ and $x_{j i_{2}}$ such that $\left(x_{j i_{1}}, x_{\overline{j i_{1}}}^{0}\right) \succ x^{0},\left(x_{j i_{2}}, x_{\overline{j i_{2}}}^{0}\right) \succ x^{0}$ and $\left(x_{j i_{1}}, x_{\overline{j i_{1}}}^{0}\right) \leftarrow x^{0} \sim_{D}\left(x_{j i_{2}}, x_{\overline{j i_{2}}}^{0}\right) \leftarrow x^{0}$. Since $X_{j I}$ is WDI (Assumption 4)

$$
\begin{gathered}
\left(x_{j i_{1}}, x_{j \bar{i}_{1}}^{0}, x_{\bar{j} I}\right) \leftarrow\left(x_{j i_{1}}^{0}, x_{j \bar{i}_{1}}^{0}, x_{\bar{j} I}\right) \sim_{D}\left(x_{j i_{2}}, x_{j \bar{j}_{2}}^{0}, x_{\bar{j} I}\right) \leftarrow\left(x_{j i_{2}}^{0}, x_{j \bar{j}_{2}}^{0}, x_{\bar{j} I}\right) \forall x_{\bar{j} I} \in X_{\bar{j} I} \\
V\left(x_{j i_{1}}, x_{j \overline{i_{1}}}^{0}, x_{\bar{j} I}\right)=V\left(x_{j i_{2}}, x_{j \bar{j}_{2}}^{0}, x_{\bar{j} I}\right) \forall x_{\bar{j} I} \in X_{\bar{j} I}
\end{gathered}
$$

First, if $V$ is multiplicative-multilinear (6), then evaluating (41) with $x_{\bar{j} I}=x_{\bar{j} I}^{0}$ gives $1+$ $\kappa v_{i_{1}}\left(x_{j i_{1}}\right) w_{i_{1}}(1)=1+\kappa w_{i_{2}}(1) v_{i_{2}}\left(x_{j i_{2}}\right)$, i.e, $w_{i_{2}}(1) v_{i_{2}}\left(x_{j i_{2}}\right)=w_{i_{1}}(1) v_{i_{1}}\left(x_{j i_{1}}\right)$. Now, if $x_{\bar{j} I}$ is equal to $x_{\bar{j} I}^{0}$ except for $k$ elements in criterion $i_{1}$, which are on the most preferred level $x_{i_{1}}^{*}$, then (41) gives $1+\kappa\left[v_{i_{1}}\left(x_{j i_{1}}\right) w_{i_{1}}(k+1)+\left(1-v_{i_{1}}\left(x_{j i_{1}}\right)\right) w_{i_{1}}(k)\right]=\left(1+\kappa w_{i_{1}}(k)\right)\left(1+\kappa w_{i_{2}}(1) v_{i_{2}}\left(x_{j i_{2}}\right)\right) \Leftrightarrow 1+$ $\kappa v_{i_{1}}\left(x_{j i_{1}}\right) w_{i_{1}}(k+1)=\left(1+\kappa w_{i_{1}}(k)\right) \kappa w_{i_{1}}(1) v_{i_{1}}\left(x_{j i_{1}}\right)+1+v_{i_{1}}\left(x_{j i_{1}}\right) w_{i_{1}}(k) \Leftrightarrow 1+\kappa w_{i_{1}}(k+1)=(1+$ $\left.\kappa w_{i_{1}}(k)\right)\left(1+\kappa w_{i_{1}}(1)\right)$, which can be used repeatedly to obtain $1+\kappa w_{i_{1}}(k+1)=\prod_{j^{\prime}=1}^{k+1}\left(1+\kappa w_{i_{1}}(1)\right)$. Substituting this into the multilinear $V_{i_{1}}(7)$ results in the multiplicative $V_{i_{1}}$, i.e., $1+\kappa V_{i_{1}}\left(x_{J i_{1}}\right)=$ $\prod_{j^{\prime}=1}^{m}\left[1+\kappa w_{i_{1}}(1) v_{i_{1}}\left(x_{j^{\prime} i_{1}}\right)\right]$ (Property (iii) of Lemma 1). Since the choice of $i_{1}$ was arbitrary, each $V_{i}$ is multiplicative and thus $V$ is multiplicative (2).

Second, if $V$ is additive-multilinear (5), then (41) gives

$$
v_{i_{1}}\left(x_{j i_{1}}\right) w_{i_{1}}(k+1)+\left(1-v_{i_{1}}\left(x_{j i_{1}}\right)\right) w_{i_{1}}(k)=w_{i_{1}}(k)+w_{i_{2}}(1) v_{i_{2}}\left(x_{j i_{2}}\right) .
$$

With $k=0$ this results in $w_{i_{2}}(1) v_{i_{2}}\left(x_{j i_{2}}\right)=w_{i_{1}}(1) v_{i_{1}}\left(x_{j i_{1}}\right)$, which can be substituted back into (42) to obtain $w_{i_{1}}(k+1)=w_{i_{1}}(k)+w_{i_{1}}(1)$. This implies that $w_{i}(k+1)=\sum_{j^{\prime}=1}^{k+1} w_{i}(1)$ for any $k=0, \ldots, m-1$ and $i \in I$. Property (ii) of Lemma 1 implies that each $V_{i}$ is linear, i.e, $V_{i}\left(x_{J i}\right)=$ $w_{i}(1) \sum_{j^{\prime}=1}^{m} v_{i}\left(x_{j^{\prime} i}\right)$, and hence $V$ is additive-linear (1).

' $\Leftarrow$ : Additive-linear and multiplicative $V$ are special cases of additive- and multiplicativemultilinear $V$, respectively, wherefore Theorem 2 implies that Assumptions 1-3 hold. The multiplicative and additive-linear $V_{i}$ can be viewed as a special cases of the general multiplicative and 
additive value functions over the $n \cdot m$ attributes and hence each subset of the attributes is WDI (Dyer and Sarin 1979). Thus for any $j \in J$, the set of attributes $X_{j I}$ is also WDI (Assumption 4).

\section{C.4. Proof of Corollary 1}

' $\Rightarrow: w_{i}(j)-w_{i}(j-1)=r_{i}\left[w_{i}(j-1)-w_{i}(j-2)\right]=r_{i}^{2}\left[w_{i}(j-2)-w_{i}(j-3)\right]=\cdots=r^{j-1}\left[w_{i}(1)-\right.$ $\left.w_{i}(0)\right]=r^{j-1} w_{i}(1)$, wherefore $w_{i}(k)=\sum_{j=1}^{k}\left[w_{i}(j)-w_{i}(j-1)\right]=\sum_{j=1}^{k} r_{i}^{j-1} w_{i}(1)$. If $r_{i}=1$, then $w_{i}(k)=\sum_{j=1}^{k} w_{i}(1)$, which can be substituted into the multilinear $V_{i}(7)$ to obtain the linear $V_{i}$ (13) (property (ii) of Lemma 1). If $r_{i} \neq 1$, then $w_{i}(k)=w_{i}(1)\left(r_{i}^{k}-1\right) /\left(r_{i}-1\right)$ and denoting $c=\left(r_{i}-1\right) / w_{i}(1)$ gives $1+c w_{i}(k)=r_{i}^{k}=\prod_{j=1}^{k} r_{i}=\prod_{j=1}^{k}\left(1+c w_{1}(1)\right)$. Substituting $1+c w_{i}(k)=$ $\prod_{j=1}^{k}\left(1+c w_{1}(1)\right)$ into the multilinear $V_{i}(7)$ gives $1+c V_{i}\left(x_{J i}\right)=\prod_{j=1}^{m}\left[1+c w_{i}(1) v_{i}\left(x_{j i}\right)\right]$ (property (iii) of Lemma 1), which is equal to the multiplicative $V_{i}(14)$.

' $\Leftarrow$ ' If $V_{i}$ is multiplicative, then $w_{i}(k)=V_{i}\left(\left\langle k, x_{i}^{*}\right\rangle\right)=w_{i}(1)\left(r_{i}^{k}-1\right) /\left(r_{i}-1\right)$ for any $k \in J$. Thus, for any $k=1, \ldots, m-1$

$$
\frac{w_{i}(k+1)-w_{i}(k)}{w_{i}(k)-w_{i}(k-1)}=\frac{r_{i}^{k+1}-r_{i}^{k}}{r_{i}^{k}-r_{i}^{k-1}}=\frac{r_{i}^{k}(r-1)}{r_{i}^{k-1}(r-1)}=r
$$

If $V_{i}$ is linear, then $w_{i}(k)=V_{i}\left(\left\langle k, x_{i}^{*}\right\rangle\right)=w_{i}(1) k$ for any $k \in J$. Thus, $w_{i}(k)-w_{i}(k-1)=w_{i}(1)$ for all $k=1, \ldots, m$.

\section{C.5. Proof of Corollary 2}

Both value functions (15) and (16) represent the preferences $x_{J i}^{a} \succeq x_{J i}^{b} \Leftrightarrow\left(\sum_{j=1}^{m} x_{j i}^{a}-x_{i}^{0}\right) \geq$ $\sum_{j=1}^{m}\left(x_{j i}^{b}-x_{i}^{0}\right)$, since they are strictly increasing in $\sum_{j=1}^{m} x_{j i}$. To show that (15) and (16) are the only functions that represent such preferences, choose arbitrary $y \in X_{i}=\left[x_{i}^{0}, x_{i}^{*}\right]$ such that $y-x_{i}^{0} \leq x_{i}^{*}-y$. Let $y^{\prime}=x^{0}+2\left(y-x^{0}\right) \leq x^{*}$. Consider two portfolios $x_{J i}^{a}=\left(y^{\prime}, x_{i}^{0}, \ldots x_{i}^{0}\right)$ and $x_{J i}^{b}=\left(y, y, x_{i}^{0}, \ldots x_{i}^{0}\right)$. Since $\sum_{j=1}^{m}\left(x_{j i}^{a}-x_{i}^{0}\right)=2\left(y-x_{i}^{0}\right)+0=\left(y-x_{i}^{0}\right)+\left(y-x_{i}^{0}\right)+0=\sum_{j=1}^{m}\left(x_{j i}^{b}-x_{i}^{0}\right)$, $x^{a} \sim x^{b}$ must hold. Evaluating the multilinear criterion specific value (7) of these portfolios gives $V_{i}\left(x_{J i}^{a}\right)=V_{i}\left(x_{J i}^{b}\right) \Leftrightarrow w_{i}(1) v_{i}\left(y^{\prime}\right)=w_{i}(2) v_{i}^{2}(y)+2 w_{i}(1)\left(1-v_{i}(y)\right) v_{i}(y) \Leftrightarrow$

$$
w_{i}(1) v_{i}\left(y^{\prime}\right)=\left[w_{i}(2)-2 w_{i}(1)\right] v_{i}^{2}(y)+2 w_{i}(1) v_{i}(y) .
$$


If $w_{i}(2)-2 w_{i}(1)=0$ then $w_{i}(2)=2 w_{i}(1)$ and $v_{i}\left(y^{\prime}\right)=2 v_{i}(y)$, which implies $v_{i}$ is linear, i.e., $v_{i}(y)=$ $\left(y-x_{i}^{0}\right) /\left(x_{i}^{*}-x_{i}^{0}\right)$. On the other hand, if $w_{i}(2)-2 w_{i}(1) \neq 0$, dividing both sides of (44) with it and denoting $D=w_{i}(1) /\left[w_{i}(2)-2 w_{i}(1)\right]$ gives $D v_{i}\left(y^{\prime}\right)=v_{i}^{2}(y)+2 D v_{i}(y)$. Adding $D^{2}$ on both sides gives $D v_{i}\left(y^{\prime}\right)+D^{2}=v_{i}^{2}(y)+2 D v_{i}(y)+D^{2} \Leftrightarrow$

$$
v_{i}\left(y^{\prime}\right)+D=\left(v_{i}(y)+D\right)^{2} / D
$$

By denoting $t=y-x^{0}$ and $g(t)=v_{i}\left(t+x^{0}\right)+D$, (45) can we written as the well-known functional equation $g(2 t)=g^{2}(t) / D$, whose solution is $g(t)=D e^{C t}$ for some $C \in \mathbb{R}$. Thus, $v_{i}(y)+D=g(y-$ $\left.x_{i}^{0}\right)=D e^{C\left(y-x^{0}\right)}$ and $C \neq 0$ (since otherwise $v_{i} \equiv 0$ ), wherefore we can denote $\rho=-1 / C$. Evaluating $v_{i}(y)=D\left(e^{\left(y-x_{i}^{0}\right) / \rho}-1\right)$ at $x_{i}^{*}$ gives the condition $v_{i}\left(x_{i}^{*}\right)=w_{i}(1)\left(e^{\left(x_{i}^{*}-x_{i}^{0}\right) / \rho}-1\right) /\left[w_{i}(2)-2 w_{i}(1)\right]=1$, which gives $w_{i}(2)=w_{i}(1)\left(1+e^{-\left(x_{i}^{*}-x_{i}^{0}\right) / \rho}\right)$ and $v_{i}(y)=\left(1-e^{-\left(y-x_{i}^{0}\right) / \rho}\right) /\left(1-e^{-\left(x_{i}^{*}-x_{i}^{0}\right) / \rho}\right)$. Thus $v_{i}$ is either linear or exponential, and fixing $w_{i}(1)$ defines a unique value for $w_{i}(2)$ in both cases. Next, we derive equations for $w_{i}(k), k=3, . ., m: \sum_{i=1}^{m}\left(x_{j i}-x_{j i}^{0}\right)=k\left(x_{i}^{*}-x_{i}^{0}\right)$ for both of the portfolios $\left\langle k, x_{i}^{*}\right\rangle$ and $\left\langle k-1, x_{i}^{*} ; 2,\left(x_{i}^{*}+x_{i}^{0}\right) / 2\right\rangle$, wherefore $V_{i}\left(\left\langle k, x_{i}^{*}\right\rangle\right)=V_{i}\left(\left\langle k-1, x_{i}^{*} ; 2,\left(x_{i}^{*}+x_{i}^{0}\right) / 2\right\rangle\right)$. Denoting $\nu=v_{i}\left(\left(x_{i}^{*}+x_{i}^{0}\right) / 2\right)$ gives

$$
\begin{aligned}
& w_{i}(k)=(1-\nu)^{2} w_{i}(k-1)+\nu(1-\nu) w_{i}(k)+\nu(1-\nu) w_{i}(k)+\nu^{2} w_{i}(k+1) \\
& -(1-\nu)^{2} w_{i}(k-1)=\nu^{2} w_{i}(k+1)+\underbrace{[-1+2 \nu(1-\nu)]}_{=-\nu^{2}-(1-\nu)^{2}} w_{i}(k) \\
& {\left[w_{i}(k)-w_{i}(k-1)\right] \frac{(1-\nu)^{2}}{\nu^{2}}=\left[w_{i}(k+1)-w_{i}(k)\right],}
\end{aligned}
$$

If $v_{i}$ is linear, then $\nu=1 / 2$ and $w_{i}(k+1)-w_{i}(k)=w_{i}(k)-w_{i}(k-1)$, wherefore the $V_{i}$ is linear (13) by Corollary 1. If $v_{i}$ is exponential, then $(1 / \nu-1)^{2}=e^{-\left(x_{i}^{*}-x_{i}^{0}\right) / \rho} \in(0, \infty)$, wherefore by Corollary $1 V_{i}$ is multiplicative (14) with $r_{i}=e^{-\left(x_{i}^{*}-x_{i}^{0}\right) / \rho}$. Substituting exponential $v_{i}\left(x_{j i}\right)=$ $\left(1-e^{-\left(x_{j i}-x_{i}^{0}\right) / \rho}\right) /\left(1-r_{i}\right)$ into $(14)$ gives

$$
\begin{aligned}
V_{i}\left(x_{J i}\right) & =\frac{w_{i}(1)}{r_{i}-1}\left[\prod_{j=1}^{m}\left(1+\left(r_{i}-1\right) \frac{1-e^{-\left(x_{j i}-x_{i}^{0}\right) / \rho}}{1-r_{i}}\right)-1\right]=\frac{w_{i}(1)}{r_{i}-1}\left[\prod_{j=1}^{m} e^{-\left(x_{j i}-x_{i}^{0}\right) / \rho}-1\right] \\
& =w_{i}(1) \frac{e^{-\sum_{j=1}^{m}\left(x_{j i}-x_{i}^{0}\right) / \rho}-1}{r_{i}-1}=w_{i}(1) \frac{1-e^{-\sum_{j=1}^{m}\left(x_{j i}-x_{i}^{0}\right) / \rho}}{1-e^{-\left(x_{i}^{*}-x_{i}^{0}\right) / \rho}}
\end{aligned}
$$

which is equal to (16). 


\section{C.6. Proof of Theorem 4}

Removing the last two lines of the Forward-loop (which may terminate the loop before $k=m$ ) results in a standard last-in-first-out type enumeration of all possible solutions $z \in\{0,1\}^{m}$ (see, e.g, Martello and Toth 1990). For each solution the algorithm checks feasibility $\left(\sum_{j=1}^{m} z_{j} a_{j} \leq b\right)$, evaluates the objective function value $V(\hat{x}(z))$ and keeps the best solution so far stored in $z^{*}$ and $V^{*}$ (solution $z=(0, \ldots, 0)^{T}$ is checked on the second line). For instance, with $m=3$ the algorithm evaluates the solutions (i.e., checks feasibility and computes $V(\hat{x}(z))$; Second line of the Forwardloop) in the following order: $z=(0,0,0), k=0 ; z=(1,0,0), k=1 ; z=(1,1,0), k=2 ; z=(1,1,1), k=$ $3 ; z=(1,0,1), k=3 ; z=(0,1,0), k=2 ; z=(0,1,1), k=3 ; z=(0,0,1), k=3$. The Forward-loop is terminated and the Backtrack-step started each time $k=m=3$, and when $z=(0,0,1), z_{m} \leftarrow 0$ results in $z=(0,0,0)$ and thus $k \leftarrow \max \left(\left\{j \in J \mid z_{j}=1\right\} \cup\{0\}\right)$ results in $k=0$, wherefore the algorithm terminates. Output $z=\emptyset$ is given if and only if there are no feasible solutions.

Next we show that the two lines that may break the Forward-loop before $k=m$ do not discard the optimal solution. Assume the algorithm is on the second line of the Forward-loop, with $k \in\{1, \ldots, m\}$ and $z \in\{0,1\}^{m}$ such that $z_{j}=0$ for all $j>k$. An arbitrary solution $z^{\prime} \in$ $\{0,1\}^{m}$ produced by continuing the Forward-loop is such that $z_{j}^{\prime}=z_{j}$ for all $j \in\{1, \ldots, k\}$. First, if $\sum_{j=1}^{k} z_{j} a_{j}+\sum_{j=k+1}^{m} \min \left\{0, a_{j}\right\}>b$ holds, then $\sum_{j=1}^{m} z_{j}^{\prime} a_{j}=\sum_{j=1}^{k} z_{j} a_{j}+\sum_{j=k+1}^{m} z_{j}^{\prime} a_{j} \geq \sum_{j=1}^{k} z_{j} a_{j}+$ $\sum_{j=k+1}^{m} \min \left\{0, a_{j}\right\}>b$, i.e., $z^{\prime}$ is infeasible and thus not the optimal solution. Second, assuming $z^{\prime}$ is feasible, for all $i \in I$,

$$
\begin{aligned}
& \sum_{j=1}^{m} v_{i}\left(\hat{x}_{j i}\left(z^{\prime}\right)\right)=\sum_{j=1}^{k} v_{i}\left(\hat{x}_{j i}(z)\right)+\sum_{j=k+1}^{m}\left[z^{\prime}{ }_{j} v_{i}\left(x_{j i}^{g o}\right)+\left(1-z^{\prime}{ }_{j}\right) v_{i}\left(x_{j i}^{n o}\right)\right] \\
& \left.\left.\leq \sum_{j=1}^{k} v_{i}\left(\hat{x}_{j i}(z)\right)+\max _{\substack{\tilde{z}_{j} \in[0,1] \\
k<j \leq m}}\left\{\sum_{j=k+1}^{m} \tilde{z}_{j} v_{i}\left(x_{j i}^{g o}\right)+\left(1-\tilde{z}_{j}\right) v_{i}\left(x_{j i}^{n o}\right) \mid \sum_{j=k+1}^{m} \tilde{z}_{j} a_{j} \leq b-\sum_{j=1}^{k} z_{j} a_{j}\right\}\right)\right\}:=s_{i},
\end{aligned}
$$

wherefore $V_{i}\left(\hat{x}_{J i}\left(z^{\prime}\right)\right) \leq \bar{V}_{i}\left(\sum_{j=1}^{m} v_{i}\left(\hat{x}_{j i}\left(z^{\prime}\right)\right)\right) \leq \bar{V}_{i}\left(s_{i}\right)$. If $g\left(\bar{V}_{1}\left(s_{1}\right), \ldots, \bar{V}_{n}\left(s_{n}\right)\right)<V^{*}$ holds, then $V\left(\hat{x}\left(z^{\prime}\right)\right)=g\left(V_{1}\left(\hat{x}_{J 1}\left(z^{\prime}\right)\right), \ldots, V_{n}\left(\hat{x}_{J n}\left(z^{\prime}\right)\right) \leq g\left(\bar{V}_{1}\left(s_{1}\right), \ldots, \bar{V}_{n}\left(s_{n}\right)\right)<V^{*}\right.$, since $g$ is strictly increasing in all its arguments. Hence $z^{\prime}$ is not the optimal solution. 


\section{C.7. Proof of Theorem 5}

By denoting $p_{j}=v_{i}\left(x_{j i}\right)$ and $g(k)=w_{i}(k)$ the multilinear $V_{i}$ becomes $V_{i}\left(x_{J i}\right)=$ $\sum_{J^{\prime} \subseteq J} g\left(\left|J^{\prime}\right|\right) \prod_{j \in J^{\prime}} p_{j} \prod_{j \notin J^{\prime}}\left(1-p_{j}\right)$. This is equivalent to computing the expectation of a random variable $g$, whose value depends on the number of successes in $m$ independent experiments with success probabilities $p=\left(p_{1}, \ldots, p_{m}\right)$. Hoeffding (1956) proves that with the expected number of successes $\sum_{j=1}^{m} p_{j}$ fixed, $\min _{p} \mathbb{E}_{p}[g]$ and $\max _{p} \mathbb{E}_{p}[g]$ are found when $p$ contains at most three different probabilities, only one of which is not equal to zero or one.

\section{Acknowledgments}

I am grateful for Antti Punkka, Alec Morton, Eeva Vilkkumaa, Ahti Salo, the area editor, the associate editor and three anonymous referees for their insightful comments and suggestions.

\section{References}

Argyris, Nikolaos, Jos Rui Figueira, Alec Morton. 2011. Interactive multicriteria methods for portfolio decision analysis. A. Salo, J. Keisler, A. Morton, eds., Portfolio Decision Analysis: Improved Methods for Resource Allocation.

Bertsimas, Dimitris, John N. Tsitsiklis. 1997. Introduction to Linear Optimization. Athena Scientific Series in Optimization and Neural Computation, 6, Athena Scientific, Belmont, MA.

Chen, Sean, Jun Liu. 1997. Statistical applications of the poisson-binomial and conditional bernoulli distributions. Statistica Sinica 7 875-892.

Clemen, R.T., J. Smith. 2009. On the choice of baselines in multiattribute portfolio analysis: A cautionary note. Decision Analysis 6(4) 256-262.

Dyer, J.S., R.K. Sarin. 1979. Measurable multiattribute value functions. Operations Research 27(4) 810-822.

Ewing Jr., P.L., W. Tarantino, G.S. Parnell. 2006. Use of decision analysis in the army base realignment and closure (brac) 2005 military value analysis. Decision Analysis 3(1) 33-49.

Golabi, K., C.W. Kirkwood, A. Sicherman. 1981. Selecting a portfolio of solar energy projects using multiattribute preference theory. Management Science 27(2) 174-189.

Grushka-Cockayne, Y., B. De Reyck, Z. Degraeve. 2008. An integrated decision-making approach for improving european air traffic management. Management Science 54(8) 1395-1409. 
Hoeffding, W. 1956. On the distribution of the number of successes in independent trials. The Annals of Mathematical Statistics 27(3) 713-721.

Keeney, R., D. von Winterfeldt. 2007. Practical value models. W. Edwards, R.F. Miles, D. von Winterfeld, eds., Advances in Decision Analysis. Cambridge University Press, 232-252.

Keeney, R.L., H. Raiffa. 1976. Decisions with Multiple Objectives: Preferences and Value Trade-Offs. John Wiley \& Sons, New York.

Kleinmuntz, D.N. 2007. Resource allocation decisions. W. Edwards, R.F. Miles, D. von Winterfeld, eds., Advances in Decision Analysis. Cambridge University Press, 400-418.

Liesiö, J., P. Mild, A. Salo. 2007. Preference programming for robust portfolio modeling and project selection. European Journal of Operational Research 181(3) 1488-1505.

Martello, S., P. Toth. 1990. Knapsack Problems - Algorithms and Computer Implementations. John Wiley \& Sons, New York.

Mild, P., A. Salo. 2009. Combining a multiattribute value function with an optimization model: An application to dynamic resource allocation for infrastructure asset management. Decision Analysis 6(3) $139-152$.

Phillips, Lawrence, Carlos Bana e Costa. 2007. Transparent prioritisation, budgeting and resource allocation with multi-criteria decision analysis and decision conferencing. Annals of Operations Research $\mathbf{1 5 4}$ $51-68$.

Salo, A., J. Keisler, A. Morton. 2011. Advances in Portfolio Decision Analysis: Improved Methods for Resource Allocation. Springer, New York.

von Winterfeldt, D., W. Edwards. 1986. Decision Analysis and Behavioral Research. Cambridge University Press. 